\title{
Geographical differentiation of apple ciders based on volatile fingerprint
}

\author{
António Sousa $^{\mathrm{a}}$, José Vareda ${ }^{\mathrm{a}}$, Regina Pereira ${ }^{\mathrm{b}}$, Catarina Silva ${ }^{\mathrm{a}}$, José S. Câmara ${ }^{\mathrm{a}, \mathrm{c}}$, \\ Rosa Perestrelo ${ }^{\mathrm{a}, *}$ \\ ${ }^{a}$ CQM - Centro de Química da Madeira, Universidade da Madeira, Campus da Penteada, 9020-105 Funchal, Portugal \\ ${ }^{\mathrm{b}}$ Direção Regional de Agricultura, Divisão de Inovação Agroalimentar, Avenida Arriaga, $n^{\circ}$ 21, Edifício Golden Gate, $9000-060$ Funchal, Portugal \\ ${ }^{\mathrm{c}}$ Departamento de Química, Faculdade de Ciências Exatas e Engenharia, Universidade da Madeira, Campus da Penteada, $9020-105$ Funchal, Portugal
}

A R T I C L E IN F O

\section{Keywords:}

Apple varieties (Malus domestica)

Volatile organic compounds

Geographical regions

Markers

\begin{abstract}
A B S T R A C T
With the globalization of food trade, it's traceability and genuineness becomes increasingly more difficult. Therefore, it is necessary to develop analytical tools to define the authenticity and genuineness of food-derived products. In the current work, headspace solid-phase microextraction followed by gas chromatography-mass spectrometry (HS-SPME/GC-MS) combined with chemometric tools was used to establish the volatile fingerprint of apple ciders produced in different geographical regions of Madeira Island, in order to define their typicity and to identify putative geographical markers. A total of 143 volatile organic compounds (VOCs) belonging to different chemical families have been identified, of which 28 were found in all apple ciders independently of geographical region. Esters, terpenic and furanic compounds presented on average a higher contribution for the total volatile fingerprint in cider produced in northern region of the Island, whereas alcohols, acids, volatile phenols, carbonyl compounds and lactones in cider from southern region. Considering the relative areas of the VOCs, 43 revealed statistically significant differences $(\mathrm{p}<0.001)$ between geographical regions, and 11 between northern and southern regions. A clear differentiation among cider-producing regions was observed on the developed partial least squares-discriminant analysis (PLS-DA) model. Two alcohols (1-hexanol, 1-octanol), 6 esters (methyl acetate, $(Z$ )-3-hexen-1-ol acetate, ethyl hexanoate, ethyl nonanote, ethyl octanoate, isoamyl octanoate) and 1 terpenic compound (limonene), can be considered putative geographic markers due to their discriminatory ability. The results obtained recognize the specific and typical geographical characteristics of the cider, which will allow the forthcoming guarantee for the construction of a sustainable platform for the establishment of the authenticity and typicality of the regional cider.
\end{abstract}

\section{Introduction}

Apple cider is a traditional alcoholic beverage fermented from apple juice, with increasing consumption and production worldwide. Apples (Malus domestica) and their derivates, particularly cider, pose a substantial impact in terms of global fruit cultivation, being the most ubiquitous and well-adapted fruit species in temperate regions (Delgado-Pelayo, Gallardo-Guerrero, \& Hornero-Méndez, 2014). In 2017, across Madeira Island, around 130 ha were dedicated to the production of 2000 tons of apples, subsequently leading to $3328 \mathrm{hl}$ of apple cider. Moreover, opposite to evidence in the remaining country, the cider-making by traditional process has never been discontinued in Madeira Island (Perestrelo, Silva, Silva, et al., 2019). The traditional cider-making started with the harvest of selected apple varieties (e.g., Azedo, Branco, Calhau, Domingos, Festa, Rijo, Ribeiro, Vime and Verde apple varieties). Then, the apples are cleaned, crushed and pressing to obtain the fruit juice without solid parts. The fruit juice is submitted to a controlled fermentation (15 to 18 days) at $18{ }^{\circ} \mathrm{C}$ to obtain an ethanol content of 7-8\% (v/v). Due to the edaphoclimatic and geographical characteristics associated with the organoleptic quality of the different endogenous varieties of cultivated apples, Madeira Island has all the natural conditions to produce excellent quality apple ciders. Based on this, the government entities encourage cider producers to maximize the potential of the cider and increase the production of apples and pears of regional varieties, which resulted in the construction of four new "sidrarias" and in the formation of a panel of tasters for the sensorial analysis of the product. The aroma and flavour of apple ciders rely on the quality of the apples used during cider-making, which is associated to several factors, namely cultivars diversity and geographical climatic conditions (Pello-Palma et al., 2017). The combination of the several factors influenced the final product with the release of volatile organic compounds (VOCs) that can be used for

\footnotetext{
* Corresponding author.

E-mail address: rmp@staff.uma.pt (R. Perestrelo).
} 
authentication purposes, having a considerable contribution on the aroma and flavour of ciders (Mangas, González, Rodríguez, \& Blanco, 1996). Therefore, the analysis of VOCs content in ciders obtained from distinct apple varieties and from different growing regions, it is crucial to achieve a high-quality product without reducing the flavour, aroma or texture (Pizarro, Pérez-del-Notario, \& González-Sáiz, 2009). In this sense, the authentication of food products is a procedure by which it is verified that the product matches the statements displayed on the label, including the raw material, the production techniques and the geographical origin (Vinci, Preti, Tieri, \& Vieri, 2012).

Typically, gas chromatography-mass spectrometry (GC-MS) combined with solid-phase microextraction (SPME) is one of the most common analytical tool used to differentiate beverages and food products from different geographical origin, through the identification of slight analytical differences on volatile fingerprints and through the establishment of putative VOCs as discriminatory geographical markers (Danezis, Tsagkaris, Camin, Brusic, \& Georgiou, 2016; Medina, Perestrelo, Santos, Pereira, \& Câmara, 2019; Perestrelo, Barros, Rocha, \& Câmara, 2014).

Regarding the research which assesses the authenticity and typicity of ciders using VOCs, Lobo et al. (Lobo, Antón-Díaz, Alonso, \& Valles, 2016) described the volatile composition and olfactometric profiles of Asturian and Basque ciders as a putative marker for origin discrimination, while Picinelli et al. (2000) differentiated Asturian ciders from foreign ones through the analysis of volatile acidity, polyalcohols and predominant VOCs. Moreover, Uthurry et al. (2019) reported the physicochemical and sensorial characteristics of ciders with the purpose to discriminate industrial and homemade ciders from the Patagonia and Cuyo regions of Argentina. Also, Qin, Petersen, and Bredie (2018) characterized the flavour profiles of commercial ciders from the UK and Scandinavian region by sensory profiling and analysis of their volatile and non-volatile components. In another case, Nicolini et al. (2018) found significant differences between the elemental composition of French and Italian Alps ciders. More recently, several studies related to the characterization of the volatile fingerprint of apple cider and juice from Madeira Island were performed by using HS-SPME/GC-MS combined with chemometric tools (e.g., principal component analysis (PCA), partial least squares - discriminant analysis (PLS-DA) (Medina et al., 2019; Perestrelo, Silva, Silva, et al., 2019). Indeed, Medina et al. (2019) established distinctive characteristics of apple juices according to Madeira Island apple varieties and geographical origin. The results showed that VOCs could be used as authenticity markers to verify variety and geographical origin of apple juices, providing local producers multiple benefits and legal protection against the misuse of products. Also, Perestrelo, Silva, Silva, et al. (2019) determined the volatile signature of apple ciders, obtained from Calhau apples (Malus domestica), from five different geographical regions of Madeira Island. A total of 107 VOCs was identified, from which 5 terpenoids, 4 alcohols, 3 acids and 3 esters presented variable importance in projection (VIP) values higher than one that could be used as putative geographical markers for cider samples.

The aim of the current work was to establish the volatile fingerprint of apple ciders obtained from Azedo, Calhau, Domingos, Festa, Golden, Marmelo, Pevide and Riscado apple varieties (Malus domestica), from different geographical origins of Madeira Island as a powerful tool to identify putative discriminatory geographical markers. In addition, compare the volatile fingerprint of apple ciders from the same apple variety and producer from two consecutive years $(2018,2019)$. To achieve the proposed goal, HS-SPME/GC-MS data combined with chemometric tools (e.g., PCA, PLS-DA) will be used. The large number of analysed samples allow to obtain a more robust PLS-DA model and an evaluation of putative geographical markers related to environmental conditions (e.g., altitude, climate). Furthermore, our study's outcomes could offer novel chances to encourage cider-making with improved content of odorants, as well as bioactive compounds, ultimately ensuring the production of high-quality products. Moreover, the results will be of outstanding importance for regional government entities to exploit the certification of regional cider as Protected Designation of Origin (PDO) and furthermore its worldwide commercialization.

\section{Materials and methods}

\subsection{Reagents and material}

Sodium chloride (NaCl, 99.5\%) was obtained from Panreac (Spain, Barcelona), and ethanol (99.9\%) was purchased from Fluka (Buchs, Switzerland). 3-Octanol, used as internal standard (IS), was obtained from Sigma-Aldrich (Madrid, Spain). The standards of VOCs, used for identification proposal, were purchased from Acros Organics (Geel, Belgium), Fluka (Buchs, Switzerland) and Sigma-Aldrich (Madrid, Spain) with high-purity grade ( $>98 \%$ ). Ultra-pure water was obtained from a Milli-Q ${ }^{\circledR}$ system (Millipore), and helium of purity 5.0, used as the GC carrier gas, was supplied from Air Liquide (Portugal). The SPME holder for manual sampling and the divinylbenzene/carboxen/polydimethylsiloxane (DVB/CAR/PDMS) fibre with 50/30 $\mu \mathrm{m}$ film thickness, $2 \mathrm{~cm}$ length fiber, were purchased from Supelco (Bellefonte, PA, USA). The $\mathrm{C}_{8}$ to $\mathrm{C}_{20}$ alkane series solution, ( $40 \mathrm{mg} / \mathrm{L}$ in n-hexane) used to calculate the kovat index (KI) was purchased from Fluka (Buchs, Switzerland).

\subsection{Cider samples}

Fifty-two apple ciders (3 samples for each lot, performing a total of 156 samples) produced in $2018(\mathrm{n}=17$, a total of 51) and 2019 ( $\mathrm{n}=35$, a total of 105) in different geographical regions of Madeira Island (Table 1) were sampled and transported to the laboratory in a cooler with ice and kept at $-80{ }^{\circ} \mathrm{C}$ until the analysis. In order, to make the discussion of the results easier, the different geographical regions were organized in two groups, based on altitude: north (Santana, São Vicente) and south (Santa Cruz, Câmara de Lobos, Calheta). In addition, apple ciders originated from the same producer in two consecutive years were considered (2018 and 2019). Sample were obtained from monovarietal apple varieties, Azedo, Calhau, Domingos, Festa, Golden, Marmelo, Pevide and Riscado (Malus domestica), according to cider producers. Briefly, the apples were washed with water for $10 \mathrm{~min}$ and constantly pressed, filtered and held in a refrigerated tank at $4{ }^{\circ} \mathrm{C}$ for $24 \mathrm{~h}$. The apple juice was diluted to a final concentration of $200 \mathrm{~g} / \mathrm{L}$ ( $23^{\circ}$ brix), and $\mathrm{SO}_{2}(80 \mathrm{mg} / \mathrm{L})$ was added. After this, $2 \%(\mathrm{v} / \mathrm{v})$ of $\mathrm{Sac}$ charomyces cerevisiae was added into the apple juice during 18 days at $18{ }^{\circ} \mathrm{C}$ in a closed fermentation tank. It should be pointed out, that the starting point for alcoholic fermentation was the same for all producers.

\subsection{Physicochemical parameters}

The $\mathrm{pH}$ of apple ciders was determined using a pH meter (FE20 Plus, Mettler-Toledo) at $22 \pm 1{ }^{\circ} \mathrm{C}$, under continuous stirring (400 rpm). The total soluble solid (TSS) was measured by using a refractometer (Atago RX-1000) at $22 \pm 1{ }^{\circ} \mathrm{C}$ and the result was expressed as ${ }^{\circ} \mathrm{Brix}$. The titratable acidity (TA) was measured by titrating with $0.1 \mathrm{M} \mathrm{NaOH}$ to a $\mathrm{pH}$ end-point with phenolphthalein $(\mathrm{pH}=8.1 \pm 0.1)$. The results were expressed as gram of malic acid per liter of apple cider ( $\mathrm{g} M A / \mathrm{L}$ ). All assays were carried out in triplicate.

\subsection{Solid phase microextraction (SPME)}

The SPME extraction conditions were adopted from a prior study carried out in our laboratory (Perestrelo, Silva, Silva, et al., 2019), with minor modifications. Shortly, in a $20 \mathrm{~mL}$ capped glass vial, $10 \mathrm{~mL}$ of sample, $10 \mu \mathrm{L}$ of 3-octanol (IS at concentration of $2.5 \mu \mathrm{g} / \mathrm{L}$ ) and $2 \mathrm{~g}$ of $\mathrm{NaCl}$ was added. Subsequently, solution was heated during $30 \mathrm{~min}$ at $50{ }^{\circ} \mathrm{C}$ in a thermostatic block, under constant stirring $(800 \mathrm{rpm})$. All 
assays were carried out in triplicate.

\subsection{Gas chromatography - Mass spectrometry (GC-MS) conditions}

The Agilent Technologies $6890 \mathrm{~N}$ Network gas chromatography was equipped with a BP20 $(30 \mathrm{~m} \times 0.25 \mathrm{~mm}$ i.d. $\times 0.25 \mu \mathrm{m})$ fused silica capillary column obtained from SGE (Darmstadt, Germany) and interfaced with an Agilent 5975 quadrupole inert mass selective detector (Palo Alto, CA, USA). After HS-SPME extraction, the fibre was introduced into the GC injector port for the thermal desorption of analytes at $250{ }^{\circ} \mathrm{C}$ during $6 \mathrm{~min}$, and a splitless injector equipped with an insert of $0.75 \mathrm{~mm}$ i.d. was used. The column flow was constant at $1 \mathrm{~mL} /$ min by using helium as a carrier gas. The following chromatographic protocol was used before the MS analysis: $40{ }^{\circ} \mathrm{C}$ increased $3{ }^{\circ} \mathrm{C} / \mathrm{min}$ to $220{ }^{\circ} \mathrm{C}$ with $10 \mathrm{~min}$ hold, for a total GC run time of $83 \mathrm{~min}$. The ion source, GC-qMS interface and quadrupole temperatures were held at 180,220 and $180^{\circ} \mathrm{C}$, respectively. MS acquisition was carried out in full scan mode $(30-300 \mathrm{~m} / z)$ with electronic impact (EI) mode at $70 \mathrm{eV}$. The electron multiplier was set to auto tune procedure. The identification of VOCs was performed by manual interpretation of spectra, by matching the mass spectra with the data system library (NIST05, Mass Search Program v.2.0d; Washington, DC) with a similarity higher than $80 \%$, by comparison with standards when available, and by KI values calculated based on the van den Dool and Kratz equation (van Den Dool \& Dec. Kratz, 1963). The KI values were compared, when available, with values reported in the literature for similar columns. The relative peak area was determined by the ratio between the GC peak areas of the VOC's peak and the internal standard's peak (IS). The relative concentration of VOCs was estimated based on the added amount of 3octanol (IS) and expressed as $\mu \mathrm{g} / \mathrm{L}$ of 3-octanol equivalents.

\subsection{Statistical analysis}

Data processing was carried out using web-based application Metaboanalyst 4.0 developed by the University of Alberta, Canada (Worley \& Powers, 2013). Prior to the statistical analysis, the data matrix was pre-processed to remove VOCs with missing values (MV), and then was normalized by cubic root and data scaling by auto-scaling. After normalization, a comparison of means of relative areas was achieved using a one-way analysis of variance (ANOVA), followed by post-hoc Tukey's test at $p$-value $<0.001$ to select the VOCs that were significant to distinguish the samples by geographical origin. Firstly, a principal component analysis (PCA), as an exploratory data analysis method, was used to visualize group tendencies based on geographical origin, using whole information contained in many variables, the VOCs fingerprint. Then, to investigate the differences in the VOCs levels of cider, PLS-DA was performed using the relative peak area of the VOCs selected by ANOVA. All the VOCs with variable importance in projection (VIP) values higher than 1.5 were considered as putative geographical markers. The efficiency and feasibility of the PLS-DA model were evaluated by $R^{2}$ (representing the goodness of fit), $Q^{2}$ (representing the predictive ability), and a permutation test (1000 permutations). The significant differences in the model were assessed by calculating the $p$-values obtained from the cross-validation analysis. For HCA, the squared Euclidean distance through Ward agglomeration method was used in the assignment of clusters.

\section{Results and discussion}

\subsection{Physicochemical parameters of apple ciders}

The physicochemical parameters of apple ciders from different geographical regions, namely $\mathrm{pH}$, total soluble solid (TSS), alcohol degree $\%$ vol. $20{ }^{\circ} \mathrm{C}$ (TAV), titratable acidity (TA) and ratio of TSS/TA are shown in Table 2. A higher $\mathrm{pH}(>4)$ value can impairs microbial stability, promotes the spoilage potential, and lead to severe flavour 
Table 2

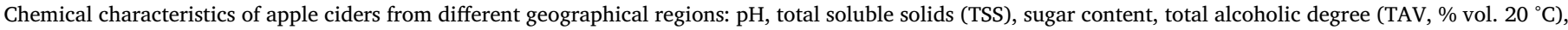
titratable acidity (TA) and ratio of TSS/TA.

\begin{tabular}{|c|c|c|c|c|c|c|c|c|c|}
\hline Province & Locality & Sample code & Apple variety & $\mathrm{pH}^{\mathrm{a}}$ & TSS $\left({ }^{\circ} \text { Brix }\right)^{\mathrm{a}}$ & Sugar $\mathbf{g} / \mathbf{L}^{\mathrm{a}}$ & TAV $(\% v o l)^{\mathrm{a}}$ & TA $(g / L)^{\mathrm{a}}$ & TSS/TA \\
\hline \multirow[t]{12}{*}{ Santana } & \multirow[t]{12}{*}{ São Roque do Faial } & A001 & Calhau & 3.55 & 17.8 & 167 & 9.92 & 8.54 & 2.08 \\
\hline & & A007_19 & Calhau & 3.53 & 15.0 & 136 & 8.08 & 7.58 & 1.98 \\
\hline & & $\mathrm{A} 002$ & Calhau & 3.57 & 16.2 & 149 & 8.86 & 7.46 & 2.17 \\
\hline & & A029_19 & Calhau & 3.41 & 13.3 & 118 & 6.99 & 7.04 & 1.89 \\
\hline & & A005 & Calhau & 3.52 & 14.5 & 131 & 7.76 & 7.85 & 1.85 \\
\hline & & A012_19 & Calhau & 3.49 & 17.4 & 163 & 9.66 & 9.01 & 1.93 \\
\hline & & A016 & Calhau & 3.34 & 14.3 & 128 & 7.63 & 8.09 & 1.77 \\
\hline & & A019_19 & Calhau & 3.40 & 14.0 & 125 & 7.43 & 7.59 & 1.84 \\
\hline & & A001_19 & Domingos & 3.45 & 17.7 & 166 & 9.86 & 6.03 & 2.94 \\
\hline & & A021_19 & Golden & 3.46 & 14.8 & 134 & 7.95 & 8.05 & 1.84 \\
\hline & & A023_19 & Domingos & 3.39 & 14.4 & 130 & 7.69 & 7.79 & 1.85 \\
\hline & & A024_19 & Festa & 3.59 & 14.6 & 132 & 7.82 & 7.06 & 2.07 \\
\hline \multirow[t]{7}{*}{ São Vicente } & \multirow[t]{5}{*}{ São Vicente } & A020 & Golden & 3.37 & 17.3 & 162 & 9.59 & 9.91 & 1.75 \\
\hline & & A002_19 & Golden & 3.35 & 16.0 & 147 & 8.73 & 7.55 & 2.12 \\
\hline & & A021 & Calhau & 3.26 & 14.9 & 135 & 8.01 & 7.08 & 2.10 \\
\hline & & A003_19 & Calhau & 3.57 & 14.1 & 126 & 7.50 & 6.34 & 2.22 \\
\hline & & A010_19 & Calhau & 3.49 & 17.4 & 163 & 9.66 & 7.59 & 2.29 \\
\hline & \multirow[t]{2}{*}{ Ponta Delgada } & A026 & Golden & 3.41 & 17.6 & 165 & 9.79 & 8.03 & 2.19 \\
\hline & & A005_19 & Golden & 3.50 & 15.7 & 144 & 8.54 & 7.67 & 2.05 \\
\hline \multirow[t]{7}{*}{ Santa Cruz } & Camacha & A006_19 & Calhau & 3.39 & 13.4 & 119 & 7.05 & 6.99 & 1.92 \\
\hline & \multirow[t]{6}{*}{ Santo da Serra } & A006 & Riscado & 3.32 & 13.2 & 117 & 6.92 & 6.56 & 2.01 \\
\hline & & A025_19 & Riscado & 3.41 & 13.7 & 122 & 7.24 & 6.48 & 2.11 \\
\hline & & A007 & Pevide & 3.42 & 15.1 & 137 & 8.15 & 7.32 & 2.06 \\
\hline & & A027_19 & Pevide & 3.28 & 13.2 & 117 & 6.92 & 7.40 & 1.78 \\
\hline & & A017 & Calhau & 3.74 & 15.6 & 143 & 8.47 & 5.76 & 2.71 \\
\hline & & A033_19 & Calhau & 3.35 & 13.8 & 123 & 7.30 & 7.32 & 1.89 \\
\hline \multirow[t]{21}{*}{ Câmara de Lobos } & \multirow[t]{21}{*}{ Jardim da Serra } & A015 & Festa & 3.42 & 14.1 & 126 & 7.50 & 5.97 & 2.36 \\
\hline & & A004_19 & Festa & 3.21 & 14.7 & 133 & 7.88 & 5.98 & 2.46 \\
\hline & & A022 & Azedo & 3.57 & 13.8 & 123 & 7.30 & 5.79 & 2.38 \\
\hline & & A009_19 & Azedo & 3.35 & 16.8 & 156 & 9.26 & 6.02 & 2.79 \\
\hline & & A024 & Domingos & 3.32 & 14.7 & 133 & 7.88 & 7.43 & 1.98 \\
\hline & & A013_19 & Domingos & 3.49 & 13.5 & 120 & 7.11 & 6.95 & 1.94 \\
\hline & & A025 & Domingos & 3.59 & 15.7 & 144 & 8.54 & 7.80 & 2.01 \\
\hline & & A015_19 & Domingos & 3.47 & 13.6 & 121 & 7.18 & 7.14 & 1.90 \\
\hline & & A028 & Domingos & 3.27 & 14.2 & 127 & 7.56 & 7.03 & 2.02 \\
\hline & & A017_19 & Domingos & 3.55 & 13.4 & 119 & 7.05 & 6.95 & 1.93 \\
\hline & & A031 & Festa & 3.28 & 15.2 & 138 & 8.21 & 7.14 & 2.13 \\
\hline & & A018_19 & Festa & 3.56 & 14.5 & 131 & 7.76 & 7.37 & 1.97 \\
\hline & & A020_19 & Festa & 3.70 & 15.1 & 137 & 8.15 & 6.69 & 2.26 \\
\hline & & A022_19 & Golden & 3.52 & 15.1 & 137 & 8.15 & 7.14 & 2.11 \\
\hline & & A026_19 & Golden & 3.51 & 12.8 & 112 & 6.66 & 7.32 & 1.75 \\
\hline & & A028_19 & Domingos & 3.48 & 13.5 & 120 & 7.11 & 7.90 & 1.71 \\
\hline & & A030_19 & Marmelo & 3.47 & 14.8 & 134 & 7.95 & 6.59 & 2.25 \\
\hline & & A031_19 & Marmelo & 3.49 & 13.3 & 118 & 6.99 & 6.78 & 1.96 \\
\hline & & A032_19 & Marmelo & 3.40 & 15.5 & 142 & 8.41 & 7.08 & 2.19 \\
\hline & & A034_19 & Calhau & 3.61 & 12.3 & 107 & 6.35 & 7.46 & 1.65 \\
\hline & & A035_19 & Calhau & 3.54 & 12.2 & 106 & 6.28 & 7.09 & 1.72 \\
\hline \multirow[t]{5}{*}{ Calheta } & \multirow[t]{3}{*}{ Prazeres } & A008 & Golden & 3.22 & 15.5 & 142 & 8.41 & 7.21 & 2.15 \\
\hline & & A008_19 & Golden & 3.49 & 13.9 & 124 & 7.37 & 7.39 & 1.88 \\
\hline & & A011_19 & Azedo & 3.45 & 12.9 & 113 & 6.73 & 7.68 & 1.68 \\
\hline & \multirow[t]{2}{*}{ Fajã da Ovelha } & A014_19 & Golden & 3.61 & 14.0 & 125 & 7.43 & 6.56 & 2.13 \\
\hline & & A016_19 & Golden & 3.65 & 13.3 & 118 & 6.99 & 6.98 & 1.91 \\
\hline
\end{tabular}

\footnotetext{
a $\%$ RSD values lower than $5 \%$ for all assays.
}

complications. A desirable apple juice $\mathrm{pH}$ range for cider-making should be from 3.2 to 3.8. On average, the highest $\mathrm{pH}$ was determined in Calhau apples $(3.49 \pm 0.10)$, whereas the lowest in Pevide apples (3.35 \pm 0.06). For the apple cider studied, the $\mathrm{pH}$ ranged from 3.21 to 3.74 , which is appropriate for the cider-making since $\mathrm{pH}$ does not exceed 4 . In ciders, the most abundant organic acids are malic acid and citric acid, representing $90 \%$ of all organic acids (Lachowicz, Oszmiański, Uździcka, \& Chmielewska, 2019), therefore the TA is usually expressed in equivalents of malic acid (MA) per liter. As observed in Table 2, on average, the lowest TA was estimated for Azedo apples $(6.50 \pm 0.23 \mathrm{~g}(\mathrm{MA}) / \mathrm{L})$, and the highest for Golden apples $(7.63 \pm 0.42 \mathrm{~g}(\mathrm{MA}) / \mathrm{L})$. The TSS was in the range from $106 \pm 3.18$ to $167 \pm 5.21 \mathrm{~g} / \mathrm{L}$, with a mean value of $133 \pm 4.36 \mathrm{~g} / \mathrm{L}$. This difference can be a result of using apple cultivar, since the Riscado $(119 \pm 1.56 \mathrm{~g} / \mathrm{L})$ and golden $(136 \pm 2.14 \mathrm{~g} / \mathrm{L})$ apple showed the lowest and the highest TSS content. The maximum TSS and the minimum TA are key parameters to the cider quality evaluation, and equally play an important role in cider stability. According to the obtained data, the ratio of TSS/TA ranged from 1.65 to 2.94, which is in agreement with the ratio reported in the literature (TSS/TA ranged from 1.5 to 3.8) (Włodarska, Pawlak-Lemańska, Górecki, \& Sikorska, 2017).

\subsection{Volatile fingerprint of apple ciders}

Fifty-two samples (156 collections) from 5 geographical regions were analysed using HS-SPME/GC-qMS methodology. Table 3 reports the frequency of occurrence (\% FO) of identified VOCs in apple cider from different geographical regions. In qualitative terms, a total of 143 VOCs were identified from a widespread of chemical families, namely 
Table 3

VOCs identified in apple ciders from different geographical regions and frequency of occurrence in percentage (\%FO).

\begin{tabular}{|c|c|c|c|c|c|c|c|}
\hline $\mathrm{RT}(\min )^{\mathrm{a}}$ & $\mathrm{KI}_{\text {cal }}{ }^{\mathrm{b}}$ & $\mathbf{K I}_{\text {Lit }}{ }^{\mathrm{c}}$ & Peak n ${ }^{\circ}$ & Chemical families & VOCs ID Id $^{\mathrm{d}}$ & North & South \\
\hline & & & & Alcohols & & & \\
\hline 13.69 & 980 & 980 & 10 & 2-Butanol & MS, ST & 21 & 30 \\
\hline 14.37 & 994 & 993 & 11 & 1-Propanol & MS, ST & 79 & 88 \\
\hline 16.91 & 1046 & 1048 & 17 & 2-Methyl-1-propanol & MS, ST & 95 & 100 \\
\hline 20.02 & 1097 & 1094 & 21 & 1-Butanol & MS, ST & 100 & 100 \\
\hline 23.58 & 1161 & 1163 & 27 & 3-Methyl-1-butanol & MS, ST & 100 & 100 \\
\hline 25.99 & 1200 & 1205 & 30 & 1-Pentanol & MS & 53 & 55 \\
\hline 29.71 & 1266 & 1268 & 41 & 2-Penten-1-ol & MS & 26 & 30 \\
\hline 29.94 & 1270 & 1280 & 42 & 2-Heptanol & MS, ST & 47 & 70 \\
\hline 30.48 & 1279 & 1282 & 45 & 4-Methyl-1-pentanol & MS & 37 & 48 \\
\hline 32.02 & 1305 & 1308 & 49 & 1-Hexanol & MS, ST & 100 & 100 \\
\hline 32.63 & 1317 & 1326 & 50 & (E)-3-Hexen-1-ol & MS, ST & 42 & 64 \\
\hline 33.87 & 1340 & 1342 & 52 & (Z)-3-Hexen-1-ol & MS, ST & 100 & 100 \\
\hline 35.55 & 1370 & 1371 & 56 & 2-Hexen-1-ol & MS & 0 & 15 \\
\hline 37.74 & 1410 & 1405 & 63 & 1-Heptanol & MS & 42 & 67 \\
\hline 38.16 & 1428 & 1428 & 64 & 1-Octen-3-ol & MS & 21 & 33 \\
\hline 39.66 & 1448 & 1446 & 70 & 2-Ethyl-1-hexanol & MS, ST & 74 & 85 \\
\hline 40.80 & 1469 & 1477 & 73 & 3-Ethyl-4-methylpentanol & MS & 47 & 48 \\
\hline 41.17 & 1476 & 1483 & 75 & 2-Nonanol & MS & 42 & 52 \\
\hline 41.47 & 1482 & 1487 & 76 & 4-Hepten-1-ol & MS & 16 & 21 \\
\hline 42.60 & 1501 & 1492 & 80 & 2,3-Butanediol & MS, ST & 37 & 18 \\
\hline 43.29 & 1509 & 1507 & 83 & 1-Octanol & MS, ST & 68 & 85 \\
\hline 46.77 & 1546 & 1536 & 87 & (E)-Hept-2-en-1-ol & MS & 21 & 36 \\
\hline 48.58 & 1624 & 1624 & 92 & 1-Nonanol & MS & 47 & 58 \\
\hline 51.85 & 1692 & 1687 & 101 & 3-(Methylthio)-1-propanol & MS, ST & 63 & 73 \\
\hline 52.02 & 1695 & 1687 & 102 & 4-Tert-butylcyclohexanol & MS & 11 & 24 \\
\hline 53.60 & 1731 & 1726 & 104 & 2-Undecanol & MS & 21 & 24 \\
\hline 54.79 & 1758 & 1760 & 106 & 1-Decanol & MS & 5 & 12 \\
\hline 56.19 & 1789 & 1789 & 110 & 1,3-Propanediol & MS & 0 & 12 \\
\hline 59.52 & 1882 & 1881 & 120 & Benzyl alcohol & MS, ST & 100 & 100 \\
\hline \multirow[t]{2}{*}{61.17} & 1907 & 1905 & 124 & 2-Phenylethyl alcohol & MS, ST & 100 & 100 \\
\hline & & & & Esters & & & \\
\hline 7.36 & 798 & 796 & 2 & Methyl acetate & MS, ST & 53 & 36 \\
\hline 8.76 & 849 & 850 & 3 & Ethyl acetate & MS, ST & 100 & 100 \\
\hline 11.10 & 918 & 910 & 6 & Ethyl propanoate & MS, ST & 84 & 73 \\
\hline 11.41 & 926 & 929 & 7 & Ethyl isobutanoate & MS & 74 & 73 \\
\hline 12.25 & 947 & 944 & 8 & Propyl acetate & MS & 32 & 24 \\
\hline 13.36 & 973 & 976 & 9 & Isobutyl acetate & MS & 84 & 82 \\
\hline 14.45 & 995 & 1000 & 12 & Ethyl butanoate & MS, ST & 100 & 100 \\
\hline 15.19 & 1014 & 1009 & 14 & Ethyl 2-methylbutanoate & MS & 100 & 100 \\
\hline 15.92 & 1028 & 1031 & 15 & Ethyl 3-methylbutanoate & MS & 100 & 100 \\
\hline 16.14 & 1032 & 1036 & 16 & Butyl acetate & MS, ST & 11 & 12 \\
\hline 18.79 & 1078 & 1080 & 19 & Propyl propanoate & MS & 95 & 100 \\
\hline 19.46 & 1089 & 1096 & 20 & Isoamyl acetate & MS, ST & 47 & 36 \\
\hline 20.92 & 1114 & 1113 & 22 & Ethyl pentanoate & MS & 26 & 15 \\
\hline 22.50 & 1143 & 1142 & 24 & Methyl hexanoate & MS & 26 & 21 \\
\hline 22.86 & 1149 & 1153 & 25 & Propyl butanoate & MS & 32 & 48 \\
\hline 25.18 & 1187 & 1183 & 28 & Ethyl hexanoate & MS, ST & 100 & 100 \\
\hline 25.52 & 1193 & 1192 & 29 & Isoamyl propanoate & MS & 37 & 33 \\
\hline 27.04 & 1219 & 1210 & 33 & Pentyl propanoate & MS & 37 & 33 \\
\hline 27.46 & 1227 & 1235 & 34 & Hexyl acetate & MS, ST & 100 & 100 \\
\hline 27.77 & 1233 & 1227 & 35 & Butyl 2-methylbutanoate & MS & 42 & 45 \\
\hline 27.94 & 1236 & 1229 & 36 & Butyl butanoate & MS & 16 & 27 \\
\hline 28.78 & 1251 & 1258 & 38 & Isoamyl butanoate & MS & 5 & 9 \\
\hline 29.18 & 1258 & 1267 & 40 & 2-Methylbutyl 2-methylbutanoate & MS & 53 & 52 \\
\hline 30.15 & 1274 & 1275 & 44 & (Z)-3-Hexen-1-ol acetate & MS, ST & 16 & 39 \\
\hline 31.01 & 1288 & 1287 & 46 & Ethyl 3-hexenoate & MS, ST & 53 & 70 \\
\hline 31.71 & 1299 & 1309 & 48 & Ethyl lactate & MS, ST & 84 & 85 \\
\hline 34.34 & 1349 & 1355 & 53 & Methyl octanoate & MS & 11 & 24 \\
\hline 36.50 & 1387 & 1389 & 60 & Ethyl 3-hydroxy-3-methylbutanoate & MS & 58 & 39 \\
\hline 36.82 & 1393 & 1392 & 61 & Ethyl octanoate & MS, ST & 100 & 100 \\
\hline 38.35 & 1432 & 1433 & 65 & Isobutyl lactate & MS & 37 & 39 \\
\hline 42.33 & 1497 & 1503 & 77 & Isoamyl hexanoate & MS, ST & 26 & 27 \\
\hline 42.86 & 1506 & 1509 & 82 & Ethyl nonanoate & MS, ST & 5 & 33 \\
\hline 44.30 & 1520 & 1527 & 84 & Ethyl 2-hydroxyhexanoate & MS & 63 & 79 \\
\hline 47.74 & 1605 & 1608 & 90 & Ethyl decanoate & MS, ST & 100 & 100 \\
\hline 48.75 & 1627 & 1625 & 93 & Isoamyl octanoate & MS & 42 & 82 \\
\hline 49.68 & 1647 & 1655 & 95 & Diethyl succinate & MS, ST & 100 & 100 \\
\hline 49.74 & 1648 & 1650 & 96 & Ethyl benzoate & MS & 0 & 15 \\
\hline 50.41 & 1662 & 1668 & 99 & Ethyl 9-decenoate & MS & 63 & 79 \\
\hline 55.40 & 1772 & 1775 & 107 & Ethyl phenylacetate & MS & 89 & 97 \\
\hline 56.89 & 1806 & 1805 & 112 & 2-Phenylethyl acetate & MS, ST & 100 & 100 \\
\hline 56.86 & 1805 & 1812 & 113 & Butyl decanoate & MS & 5 & 9 \\
\hline 57.63 & 1828 & 1827 & 115 & Ethyl dodecanoate & MS, ST & 100 & 100 \\
\hline
\end{tabular}


Table 3 (continued)

\begin{tabular}{|c|c|c|c|c|c|c|c|}
\hline RT $(\min )^{\mathrm{a}}$ & $\mathrm{KI}_{\text {cal }}{ }^{\mathrm{b}}$ & $\mathrm{KI}_{\text {Lit }}{ }^{\mathrm{c}}$ & Peak $\mathbf{n}^{\circ}$ & Chemical families & VOCs ID $^{\mathrm{d}}$ & North & South \\
\hline 58.53 & 1854 & 1864 & 118 & Ethyl 3-hydroxydodecanoate & MS & 16 & 36 \\
\hline 59.92 & 1894 & 1895 & 121 & Docyl acetate & MS & 11 & 30 \\
\hline 60.07 & 1885 & 1886 & 122 & Ethyl benzenepropanoate & MS & 37 & 36 \\
\hline 60.41 & 1892 & 1897 & 123 & Propyl dodecanoate & MS & 100 & 100 \\
\hline 62.31 & 1931 & 1923 & 125 & Benzyl benzoate & MS & 26 & 6 \\
\hline 62.42 & 1932 & 1926 & 126 & Ethyl tridecanoate & MS & 21 & 33 \\
\hline 66.65 & 2017 & 2023 & 132 & Diethyl dl-malate & MS & 11 & 36 \\
\hline 70.75 & 2099 & 2094 & 134 & Ethyl tetradecanoate & MS, ST & 63 & 70 \\
\hline 74.92 & 2126 & 2126 & 137 & Ethyl pentadecanoate & MS, ST & 100 & 100 \\
\hline 77.69 & 2209 & 2215 & 139 & Ethyl hexadecanoate & MS, ST & 37 & 39 \\
\hline 79.87 & 2218 & 2221 & 143 & $\begin{array}{l}\text { Hexyl salicylate } \\
\text { Acids }\end{array}$ & MS & 5 & 9 \\
\hline 39.65 & 1447 & 1448 & 69 & Acetic acid & MS, ST & 79 & 73 \\
\hline 45.56 & 1534 & 1533 & 85 & 2-Methylpropanoic acid & MS & 16 & 24 \\
\hline 50.16 & 1657 & 1644 & 97 & 2-Methylbutanoic acid & MS & 32 & 36 \\
\hline 59.02 & 1868 & 1868 & 119 & Hexanoic acid & MS, ST & 100 & 100 \\
\hline 62.89 & 1942 & 1943 & 127 & 2-Ethylhexanoic acid & MS, ST & 5 & 24 \\
\hline 68.08 & 2046 & 2046 & 133 & Octanoic acid & MS, ST & 100 & 100 \\
\hline 76.79 & 2205 & 2206 & 138 & 4-Ethyloctanoic acid & MS, ST & 100 & 100 \\
\hline 79.78 & 2218 & 2227 & 142 & $\begin{array}{l}\text { Decanoic acid } \\
\text { Carbonyl compounds }\end{array}$ & $\mathrm{MS}, \mathrm{ST}$ & 16 & 39 \\
\hline 5.58 & 721 & 723 & 1 & Acetaldehyde & MS, ST & 89 & 73 \\
\hline 9.17 & 862 & 866 & 4 & 2-Butanone & MS, ST & 11 & 18 \\
\hline 9.72 & 879 & 884 & 5 & 3-Methyl-butanal & MS, ST & 32 & 33 \\
\hline 22.32 & 1139 & 1141 & 23 & 3-Heptanone & MS, ST & 21 & 39 \\
\hline 26.52 & 1210 & 1210 & 31 & 3-Octanone & MS, ST & 32 & 39 \\
\hline 28.61 & 1248 & 1248 & 37 & Octanal & MS, ST & 11 & 21 \\
\hline 28.82 & 1254 & 1258 & 39 & 3-Hydroxy-2-butanone & MS, ST & 26 & 30 \\
\hline 31.44 & 1295 & 1301 & 47 & 6-Methyl-5-hepten-2-one & MS & 16 & 18 \\
\hline 34.37 & 1350 & 1350 & 54 & 2-Nonanone & MS, ST & 11 & 18 \\
\hline 34.98 & 1360 & 1361 & 55 & Nonanal & MS, ST & 53 & 76 \\
\hline 36.24 & 1383 & 1389 & 58 & 2,4-Hexadienal & MS & 47 & 64 \\
\hline 40.59 & 1465 & 1469 & 72 & Decanal & MS, ST & 58 & 55 \\
\hline 42.36 & 1498 & 1486 & 78 & Benzaldehyde & MS, ST & 21 & 42 \\
\hline 56.81 & 1803 & 1803 & 111 & Tridecanal & MS & 11 & 24 \\
\hline 57.71 & 1830 & 1831 & 116 & 4-Propyl-benzaldehyde & MS & 58 & 70 \\
\hline 79.16 & 2215 & 2219 & 140 & $\begin{array}{l}\text { Hexadecenal } \\
\text { Terpenic compounds }\end{array}$ & MS & 5 & 6 \\
\hline 23.11 & 1153 & 1153 & 26 & Limonene & MS, ST & 47 & 9 \\
\hline 35.87 & 1376 & 1388 & 57 & (E)-Linalool oxide & MS & 26 & 33 \\
\hline 38.96 & 1434 & 1428 & 67 & (Z)-Linalool oxide & MS & 16 & 36 \\
\hline 42.79 & 1504 & 1506 & 81 & Linalool & MS, ST & 37 & 21 \\
\hline 46.15 & 1540 & 1547 & 86 & 4-Terpineol & MS & 58 & 64 \\
\hline 47.46 & 1554 & 1563 & 89 & $\beta$-Terpineol & MS, ST & 21 & 6 \\
\hline 50.86 & 1672 & 1669 & 100 & $\alpha$-Terpeniol & MS, ST & 95 & 100 \\
\hline 53.25 & 1723 & 1730 & 103 & $\alpha$-Curcumene & MS & 37 & 24 \\
\hline 55.59 & 1776 & 1783 & 109 & Geraniol & MS, ST & 5 & 15 \\
\hline 66.23 & 2009 & 2008 & 130 & $\begin{array}{l}\text { Nerolidol } \\
\mathbf{C}_{\mathbf{1 3}} \text { Norisoprenoids }\end{array}$ & MS & 100 & 100 \\
\hline 57.22 & 1815 & 1814 & 114 & $\beta$-Damascenone & MS, ST & 100 & 100 \\
\hline 58.40 & 1850 & 1848 & 117 & $\begin{array}{l}\text { Geranyl acetone } \\
\text { Furanic compounds }\end{array}$ & MS, ST & 63 & 76 \\
\hline 36.25 & 1383 & - & 59 & Tetrahydro-2,5-dimethoxyfuran & MS & 16 & 30 \\
\hline 38.83 & 1431 & 1431 & 66 & 2-Furfural & MS, ST & 11 & 15 \\
\hline 47.23 & 1551 & 1553 & 88 & Methyl 2-furoate & MS, ST & 11 & 18 \\
\hline 48.88 & 1630 & 1623 & 94 & $\begin{array}{l}\text { 2-Furanmethanol } \\
\text { Lactones }\end{array}$ & MS & 5 & 6 \\
\hline 48.11 & 1613 & 1611 & 91 & Butyrolactone & MS, ST & 42 & 36 \\
\hline 63.68 & 1958 & 1957 & 129 & $\begin{array}{l}(Z) \text {-Wiskey lactone } \\
\text { Volatile phenols }\end{array}$ & MS, ST & 11 & 21 \\
\hline 63.21 & 1945 & 1945 & 128 & 4-Methyl guaiacol & MS & 0 & 9 \\
\hline 66.42 & 2012 & 2010 & 131 & 4-Ethyl guaiacol & MS, ST & 100 & 100 \\
\hline 72.06 & 2108 & 2116 & 135 & Eugenol & MS, ST & 100 & 100 \\
\hline 72.25 & 2109 & 2110 & 136 & 4-Ethyl phenol & MS, ST & 100 & 100 \\
\hline 79.23 & 2218 & 2218 & 141 & $\begin{array}{l}\text { 4-Vinylguaiacol } \\
\text { Miscellaneous }\end{array}$ & MS & 32 & 27 \\
\hline 14.77 & 1005 & 1011 & 13 & Toluene & MS, ST & 11 & 33 \\
\hline 17.76 & 1061 & 1070 & 18 & 2-Ethyl anisole & MS & 68 & 48 \\
\hline 26.84 & 1216 & 1224 & 32 & Styrene & MS & 100 & 100 \\
\hline 30.13 & 1273 & 1269 & 43 & 4-Ethyl toluene & MS & 21 & 9 \\
\hline 33.36 & 1330 & 1335 & 51 & 2-Ethyl p-xylene & MS & 32 & 42 \\
\hline 37.32 & 1401 & 1400 & 62 & 1,2,4,5-Tetramethyl-benzene & MS & 47 & 64 \\
\hline 39.64 & 1447 & 1446 & 68 & 3-Methylanisole & MS & 16 & 27 \\
\hline 40.35 & 1461 & 1456 & 71 & $1,2,3,5$-Tetramethyl-benzene & MS & 21 & 45 \\
\hline 41.07 & 1474 & 1468 & 74 & 4-Methylindan & MS & 5 & 12 \\
\hline
\end{tabular}


Table 3 (continued)

\begin{tabular}{|c|c|c|c|c|c|c|c|}
\hline RT $(\min )^{\mathrm{a}}$ & $\mathrm{KI}_{\mathrm{cal}}^{\mathrm{b}}$ & $\mathrm{KI}_{\text {Lit }}{ }^{\mathrm{c}}$ & Peak $\mathbf{n}^{\circ}$ & Chemical families & VOCs ID ${ }^{\mathrm{d}}$ & North & South \\
\hline 42.39 & 1499 & 1501 & 79 & 4-Ethylanisole & MS & 11 & 18 \\
\hline 50.21 & 1658 & 1660 & 98 & 4-Vinylanisole & MS & 37 & 33 \\
\hline 53.75 & 1735 & 1730 & 105 & Naphthalene & MS & 100 & 97 \\
\hline 55.40 & 1772 & 1771 & 108 & 2-Acetylthiophene & MS & 26 & 3 \\
\hline
\end{tabular}

-: Not detected.

a Retention time (min) determined in a BP-20 capillary column.

b Kovat index relative n-alkanes $\left(\mathrm{C}_{8}\right.$ to $\mathrm{C}_{20}$ ) on a BP-20 capillary column.

c Kovat index relative reported in literature for equivalent capillary column in Pubmed database.

d VOCs identification using mass spectra of the NIST database (MS) and standard (ST).
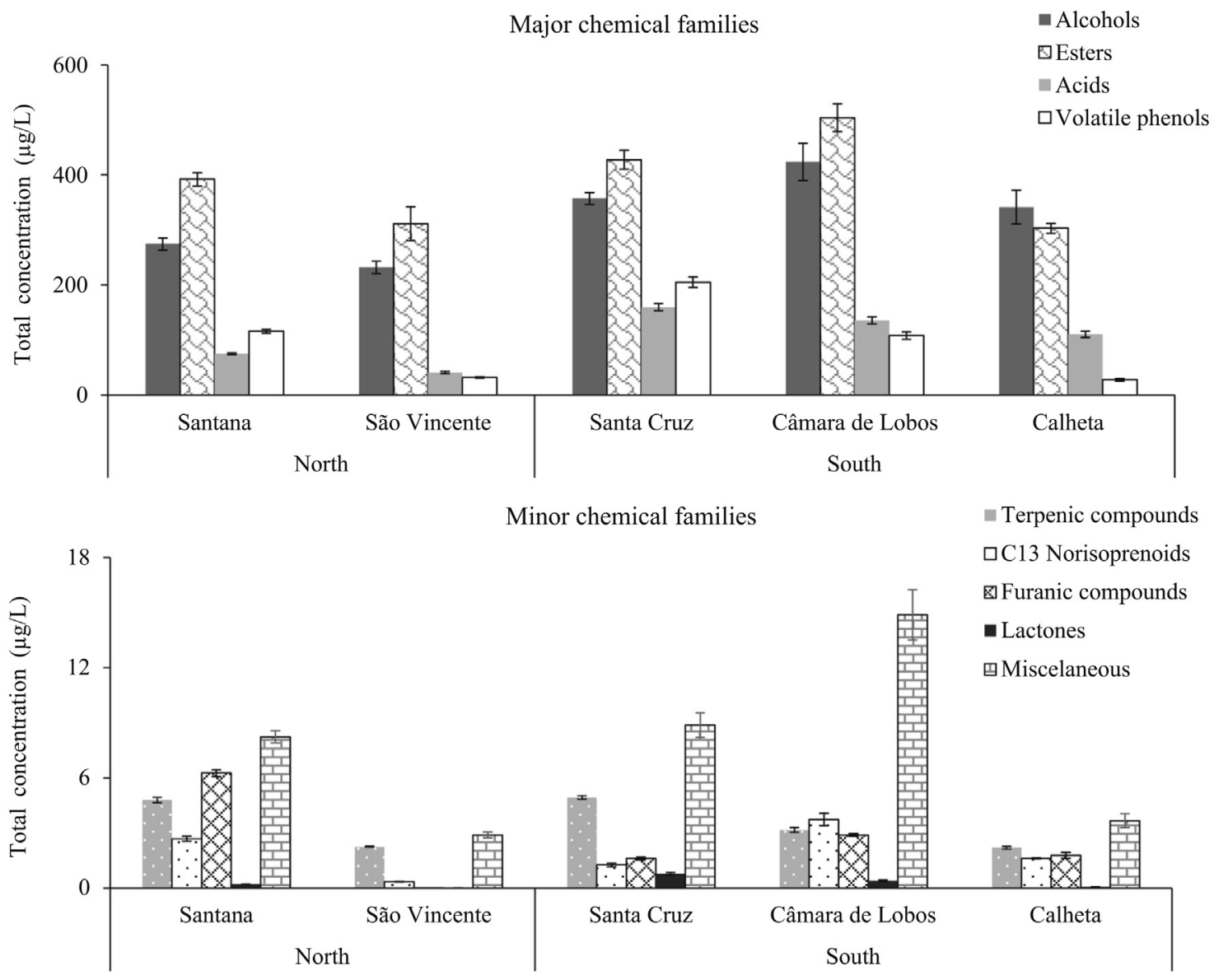

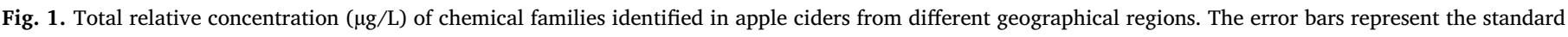
deviation for each measurement $(n=3)$.

30 alcohols, 53 esters, 8 acids, 16 carbonyl compounds, 10 terpenic compounds, $2 \mathrm{C}_{13}$ norisoprenoids, 4 furanic compounds, 2 lactones, 5 volatile phenols, and 13 miscellaneous. As many as 28 of the 143 identified VOCs showed a FO of 100\%, namely 6 alcohols, 13 esters, 3 acids, 1 terpenic compounds, $1 \mathrm{C}_{13}$ norisoprenoid, 3 volatile phenols and 1 miscellaneous. Four of VOCs were identified only in apple ciders produced in southern regions of Madeira Island, namely 2-hexen-1-ol, 1,3-propanediol, ethyl benzoate and 4-methyl guaiacol. On the other hand, 2-penten-1-ol, (E)-hept-2-en-1-ol, 1-nonanol, butyl acetate, isoamyl propanoate, ethyl 3-hexenoate, ethyl nonoate, butyl decanoate, docyl acetate, ethyl tridecanoate, ethyl hexadecanoate, 2-methylpropanoic acid, 2-ethylhexanoic acid, decanoic acid, 3-octanone, 3-hydroxy-2-butanone, nonanal, tetrahydro-2,5-dimethoxyfuran and toluene have not been identified in São Vicente apple ciders, whereas 2,3butandiol, propyl acetate, octanal and $\beta$-terpineol were not identified in the apple ciders produced in Calheta. A high number $\mathrm{f}$ these VOCs have been reported earlier in different cider types, as well as other cider derivatives (Lobo et al., 2016; Medina et al., 2019; Nicolini et al., 2018; Perestrelo, Silva, Silva, et al., 2019; Picinelli et al., 2000).

In semi-quantitative terms, esters (on average $381 \pm 18.8 \mu \mathrm{g} / \mathrm{L}$ of the total relative concentration of volatile fingerprint), alcohols (on average $313 \pm 21.5 \mu \mathrm{g} / \mathrm{L})$, acids $(96.3 \pm 4.52 \mu \mathrm{g} / \mathrm{L})$, volatile phenols $(93.5 \pm 2.60 \mu \mathrm{g} / \mathrm{L})$ and carbonyl compounds $(15.0 \pm 1.03 \mu \mathrm{g} / \mathrm{L})$ were the chemical families with the highest contribution to the volatile fingerprint of the investigated apple ciders (Fig. 1). The contribution of the remaining chemical families for the total volatile fingerprint was lower than $1 \%$.

The esters were the major chemical family of VOCs, contributing with $46.7 \pm 2.04$ and $38.4 \pm 1.56 \%$ of the total volatile fingerprint of apple ciders produced in the north and south of Madeira Island. Apple ciders from Câmara de Lobos seem to be the richest in esters $(504 \pm 20.7 \mu \mathrm{g} / \mathrm{L})$, followed by apple ciders produced in Santa Cruz 
Table 4

Important volatile organic compounds identified by t-tests $(p<0.001)$ between northern and southern Madeira Island regions.

\begin{tabular}{lllll}
\hline VOCs & t stat & p value & $-\log 10(\mathrm{p})$ & FDR \\
\hline Isoamyl octanoate & -5.52 & $1.38 \mathrm{E}-07$ & 6.86 & $5.80 \mathrm{E}-06$ \\
Ethyl octanoate & -4.99 & $1.65 \mathrm{E}-06$ & 5.78 & $3.43 \mathrm{E}-05$ \\
Methyl acetate & 4.90 & $2.45 \mathrm{E}-06$ & 5.61 & $3.43 \mathrm{E}-05$ \\
Limonene & 4.60 & $8.67 \mathrm{E}-06$ & 5.06 & $6.68 \mathrm{E}-05$ \\
Ethyl nonanoate & -4.59 & $9.01 \mathrm{E}-06$ & 5.05 & $6.68 \mathrm{E}-05$ \\
1,2,4,5-Tetramethyl-benzene & -4.56 & $1.02 \mathrm{E}-05$ & 4.99 & $6.68 \mathrm{E}-05$ \\
1-Hexanol & -4.54 & $1.11 \mathrm{E}-05$ & 4.95 & $6.68 \mathrm{E}-05$ \\
Ethyl hexanoate & -4.48 & $1.43 \mathrm{E}-05$ & 4.84 & $7.51 \mathrm{E}-05$ \\
1-Octanol & -4.36 & $2.41 \mathrm{E}-05$ & 4.62 & $1.12 \mathrm{E}-04$ \\
(Z)-3-Hexen-1-ol acetate & -4.13 & $5.96 \mathrm{E}-05$ & 4.22 & $2.50 \mathrm{E}-04$ \\
Benzyl benzoate & 4.08 & $7.27 \mathrm{E}-05$ & 4.14 & $2.77 \mathrm{E}-04$ \\
Nonanal & -4.00 & $9.72 \mathrm{E}-05$ & 4.01 & $3.40 \mathrm{E}-04$ \\
Octanoic acid & -3.90 & $1.42 \mathrm{E}-04$ & 3.85 & $4.51 \mathrm{E}-04$ \\
Ethyl 2-hydroxyhexanoate & -3.89 & $1.50 \mathrm{E}-04$ & 3.82 & $4.51 \mathrm{E}-04$ \\
\hline
\end{tabular}

FDR - False discover rate.

(427 $\pm 25.3 \mu \mathrm{g} / \mathrm{L})$, Santana (392 $\pm 19.4 \mu \mathrm{g} / \mathrm{L})$, São Vicente $(311 \pm 12.9 \mu \mathrm{g} / \mathrm{L})$ and Calheta $(303 \pm 15.9 \mu \mathrm{g} / \mathrm{L})$. They are formed by yeasts during fermentation and can impart a pleasant flavour like fruits and floral in overall cider aroma. Within ciders, ethyl esters derived from ethanol (e.g., ethyl butanoate, ethyl hexanoate, ethyl octanoate), and acetates derived from acetic acid (e.g., ethyl acetate, hexyl acetate) are the dominant VOCs in this chemical family (Nešpor, Karabín, Štulíková, \& Dostálek, 2019). Ethyl acetate (on average, the relative concentration in apple cider produced in northern and southern of Madeira Island was $113 \pm 2.63$ and $95.4 \pm 3.45 \mu \mathrm{g} / \mathrm{L}$, respectively) is the most predominant ester, followed, in lower, extent by ethyl octanoate $(66.7 \pm 1.96$ and $110 \pm 6.14 \mu \mathrm{g} / \mathrm{L})$, ethyl hexanoate $(24.7 \pm 0.96$ and $42.2 \pm 1.27 \mu \mathrm{g} / \mathrm{L})$ and ethyl decanoate $(31.1 \pm 1.78$ and $34.6 \pm 2.97 \mu \mathrm{g} / \mathrm{L})$. From these, ethyl octanoate and ethyl hexanoate showed significant differences $(\mathrm{p}<0.001)$ between northern and southern of Madeira Island regions (Table 4). The relative concentration of ethyl hexanoate and ethyl octanoate was above of its odour threshold (OT $=14$ and $5 \mu \mathrm{g} / \mathrm{L}$ determined in water $/ 10 \%$ ethanol solution at pH 3.2 (Mestre et al., 2019), respectively). Consequently these VOCs can contribute positively with fruits, apple, pear, and pineapple notes to sensorial properties of apple ciders, as well as might probably be used as discriminant markers. Moreover, Antón, Suárez Valles, García Hevia, and Picinelli Lobo (2014) reported ethyl hexanoate and ethyl octanoate as putative VOC odorants of Asturias cider.

Alcohols are formed through $\alpha$-keto acids produced either anabolically via the biosynthetic route from the carbon source, or catabolically, via the degradation of amino acids (Ehrlich pathway) (Sun et al., 2019). The occurrence of this chemical family at concentrations higher than $400 \mathrm{mg} / \mathrm{L}$ can impart a strong, pungent smell and taste. However, if lower than $300 \mathrm{mg} / \mathrm{L}$ can contribute positively to the overall aroma with a fruity character (González-Robles \& Cook, 2016). The alcohols contribution for the total volatile fingerprint is similar among the apple ciders in the north and south of Madeira Island, $33.8 \pm 1.98$ and $35.8 \pm 2.86 \%$, respectively. The alcohols' contribution is strongly influenced by 3-methylbutan-1-ol (on average, the relative peak area in apple cider produced in northern and southern of Madeira Island was
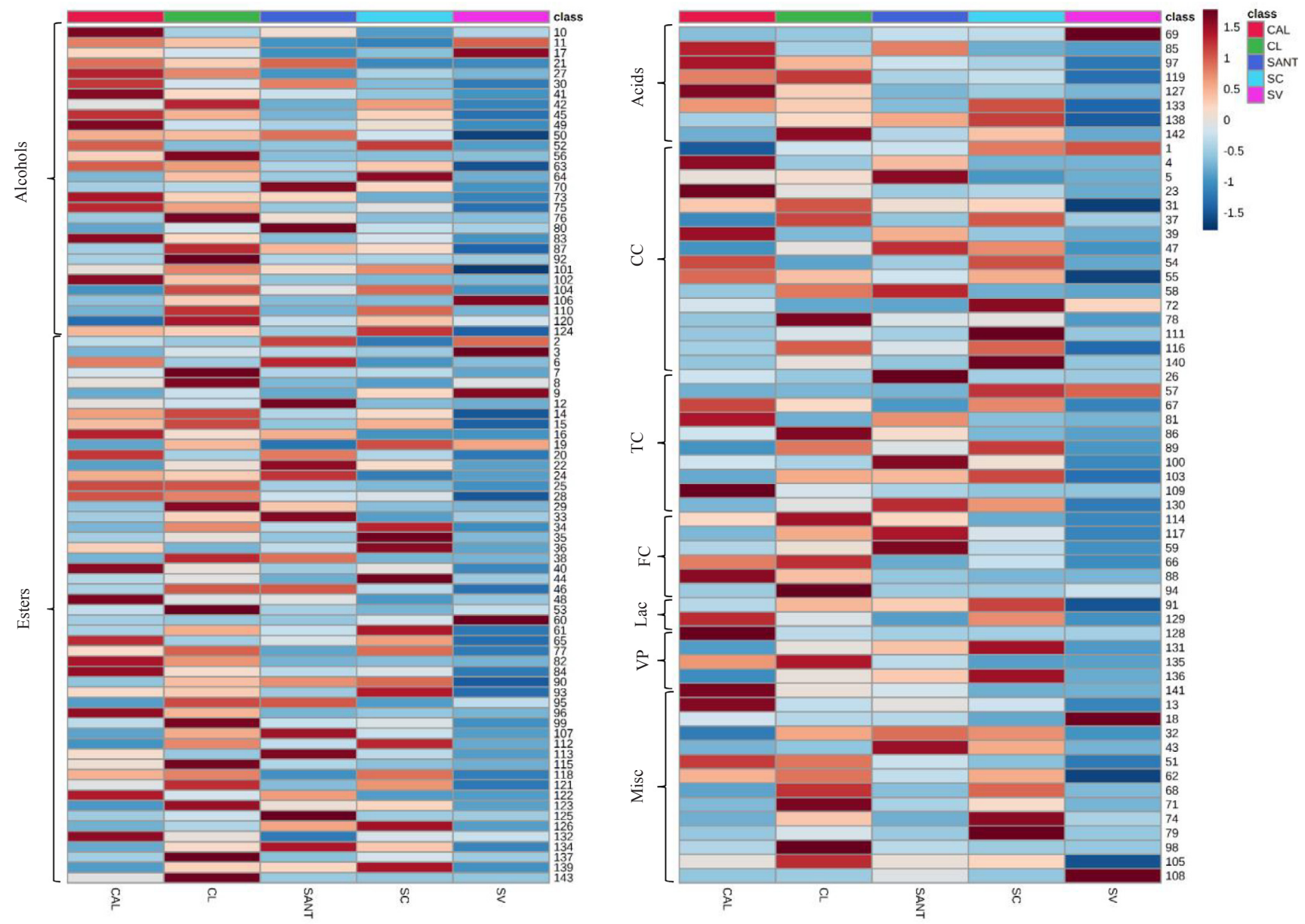

Fig. 2. Heatmap of the mean relative concentration of volatile organic compounds identified in apple ciders by geographical regions (peak number attribution is shown in Table 3). CC - Carbonyl compounds; TC - Terpenic compounds; FC - Furanic compounds; Lac - Lactones; VP - Volatile phenols; Misc - Miscellaneous. 


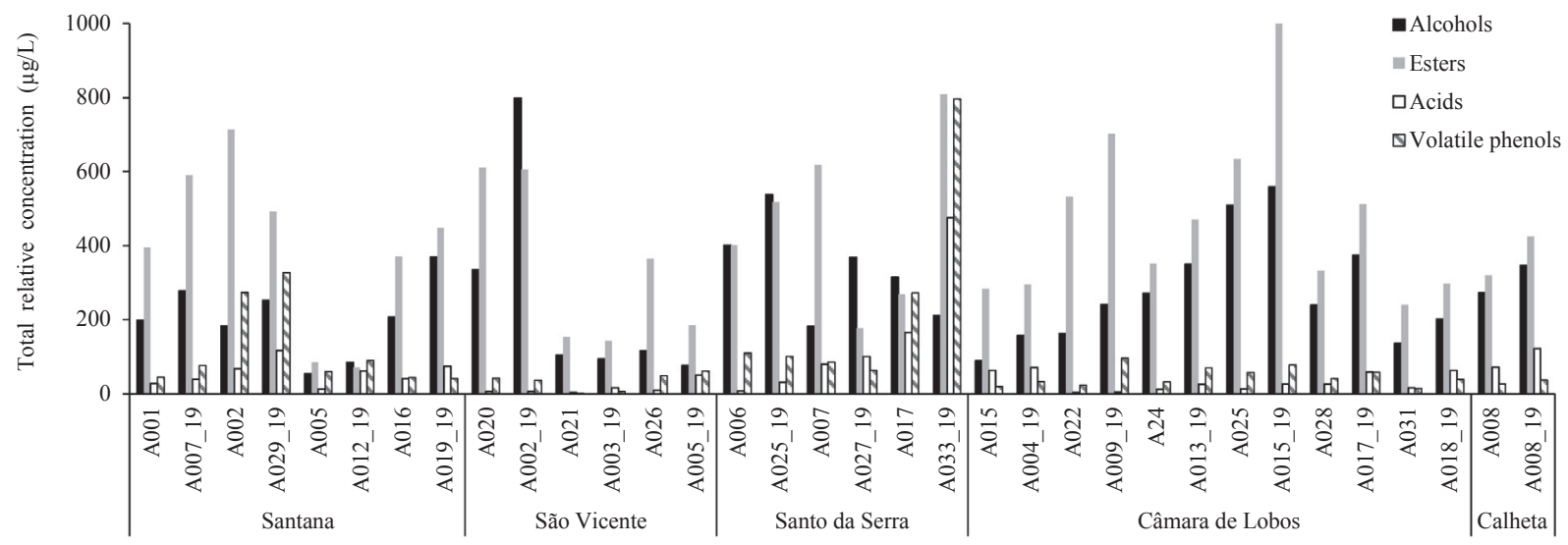

Fig. 3. Comparison of total relative concentration $(\mu \mathrm{g} / \mathrm{L})$ by major chemical families identified in apple ciders produced in 2018 and 2019.

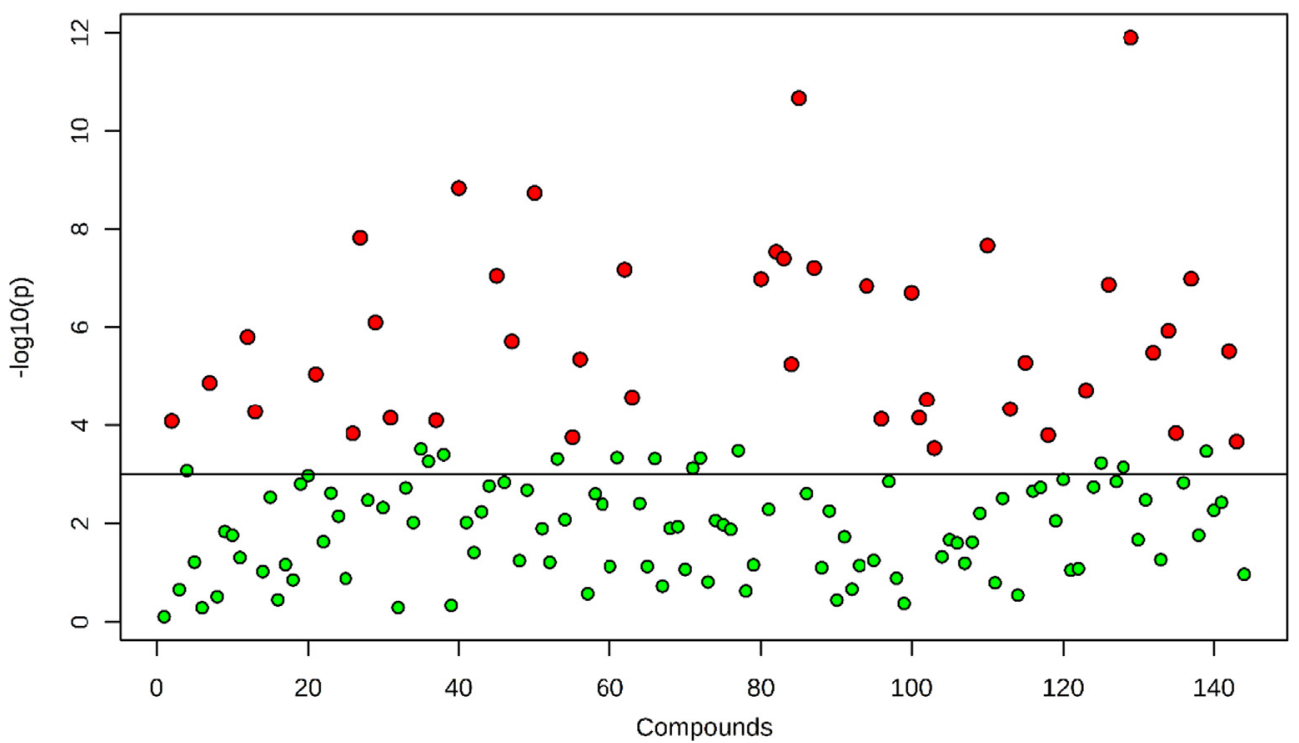

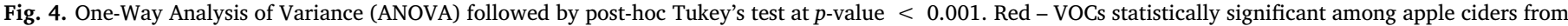
different geographical regions. (For interpretation of the references to colour in this figure legend, the reader is referred to the web version of this article.)

$147 \pm 10.3$ and $260 \pm 24.6 \mu \mathrm{g} / \mathrm{L}$, respectively), followed by phenylethyl alcohol (51.4 \pm 2.09 to $68.3 \pm 1.46 \mu \mathrm{g} / \mathrm{L})$, 1 -hexanol $(29.5 \pm 2.14$ to $36.8 \pm 3.07)$ and 2-methyl-1-propanol (16.2 \pm 1.96 and $15.7 \pm 1.06 \mu \mathrm{g} / \mathrm{L}$ ). 3-Methyl-1-butanol and 2-phenylethyl alcohol are widely reported in fermented beverages, including ciders (Antón et al., 2014; Perestrelo, Silva, Silva, et al., 2019), beers (Andrés-Iglesias, Blanco, García-Serna, Pando, \& Montero, 2016) and wines (Perestrelo, Silva, \& Câmara, 2019), to which are associated by banana and rose-like odours, respectively. Nonetheless, the relative concentration of 3-methyl-1-butanol and 2-phenylethyl alcohol was below of their OTs, 30,000 and $14000 \mu \mathrm{g} / \mathrm{L}$ (Mestre et al., 2019), respectively. Therefore, these alcohols cannot influence the apple cider sensorial properties. 1Hexanol is a typical VOC from apple fruits and is formed during the enzymatic oxidation process of linoleic acid (Medina et al., 2019), and showed significant differences $(\mathrm{p}<0.001$ ) between northern and southern of Madeira Island regions (Table 3). Nonetheless, the relative concentration of 1-hexanol in apple cider was below the value of its OT ( $2500 \mu \mathrm{g} / \mathrm{L}$ (Mestre et al., 2019)). So, this VOC probably will be used as discriminant marker but no as compound of influence for sensorial properties.

Acids are formed through the metabolism of lipids by yeast. At concentrations higher than their odour thresholds typically imparts unpleasant flavours (e.g., fatty, sweat, rancid, cheese) (Tesnière, 2019). The contribution of this chemical family to the total volatile fingerprint of apple cider produced in northern and southern regions of Madeira Island is significantly different, $7.42 \pm 0.26$ and $12.8 \pm 0.78 \%$, respectively. The high relative concentration of octanoic $(27.1 \pm 0.97$ and $72.4 \pm 6.89 \mu \mathrm{g} / \mathrm{L}$ for northern and southern regions of Madeira Island, respectively) and decanoic (15.9 \pm 2.05 and $30.6 \pm 1.13 \mu \mathrm{g} /$ L) acids explains the difference observed in apple ciders from northern and southern regions. Their ratio, between northern and southern, is almost 2.68 and 1.92 times higher, respectively. Nevertheless, since their relative concentration was below their OTs, 3000 and $10000 \mathrm{mg} / \mathrm{L}$ (Mestre et al., 2019), respectively, any influence on apple cider sensorial properties is no expected. Moreover, 2-methylpropanoic, 2ethylhexanoic and decanoic acids were not detected in any of apple ciders produced in São Vicente.

Volatile phenols are produced by decarboxylation of hydroxycinnamic acids (Lentz, 2018), and represent $8.98 \pm 0.49$ and $9.84 \pm 0.56 \%$ of the total volatile fingerprint of apple ciders produced in the northern and southern regions of Madeira Island, respectively. Apple ciders from Santa Cruz seem to be the richest in volatile phenols $(205 \pm 20.6 \mu \mathrm{g} / \mathrm{L})$, followed by apple ciders produced in Santana $(115 \pm 2.98 \mu \mathrm{g} / \mathrm{L})$, Câmara de Lobos (108 $\pm 1.79 \mu \mathrm{g} / \mathrm{L})$, São Vicente $(31.7 \pm 1.09 \mu \mathrm{g} / \mathrm{L})$ and Calheta $(27.6 \pm 0.98 \mu \mathrm{g} / \mathrm{L})$. From the pool of volatile phenols identified, 4-ethyl guaiacol (the average relative peak area in apple cider produced in northern and southern regions of Madeira Island was $35.4 \pm 1.73$ and $33.6 \pm 2.75 \mu \mathrm{g} / \mathrm{L}$, respectively) and 


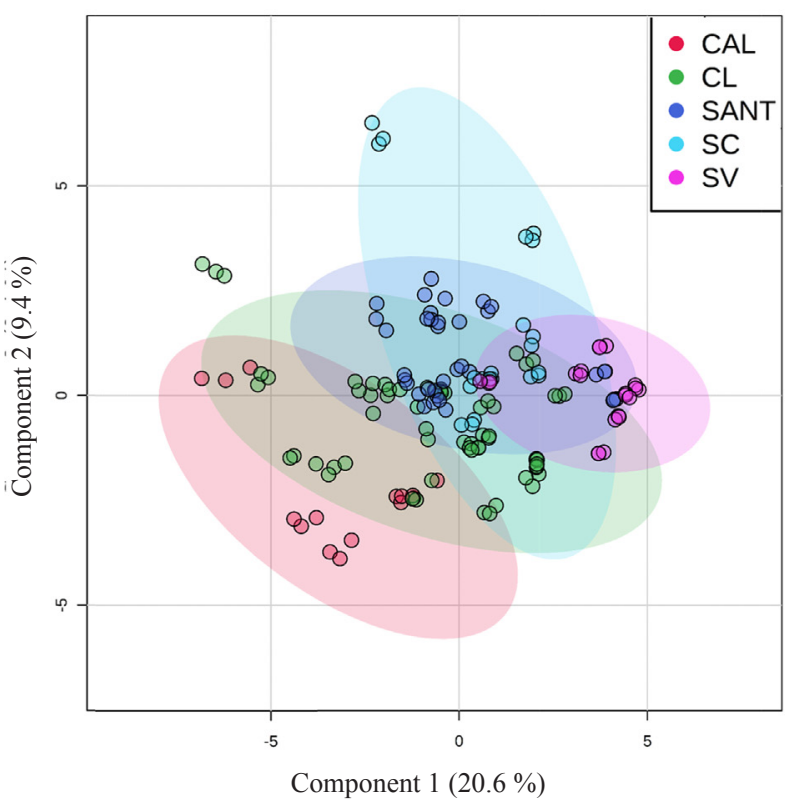

(a)

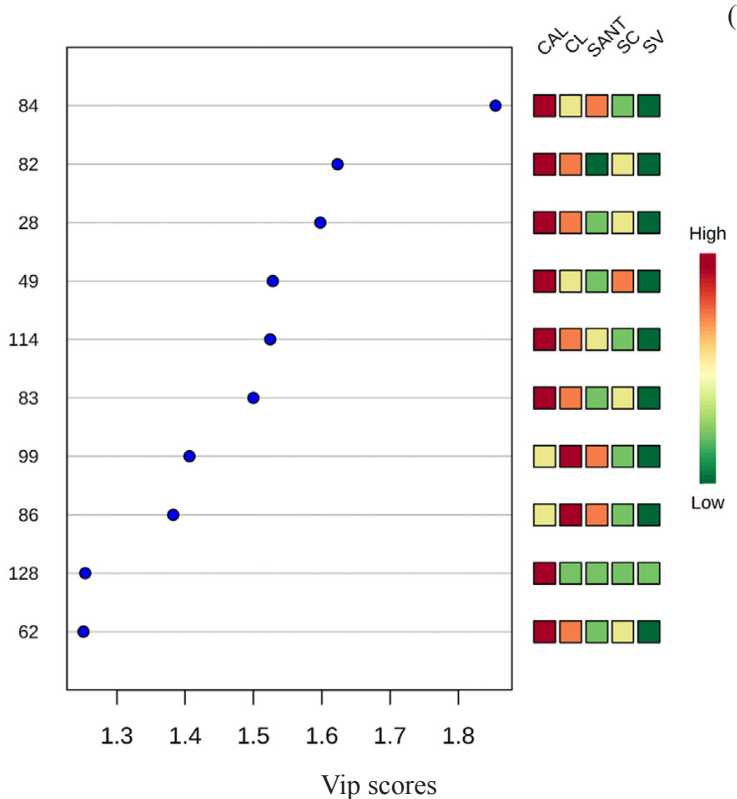

(b)
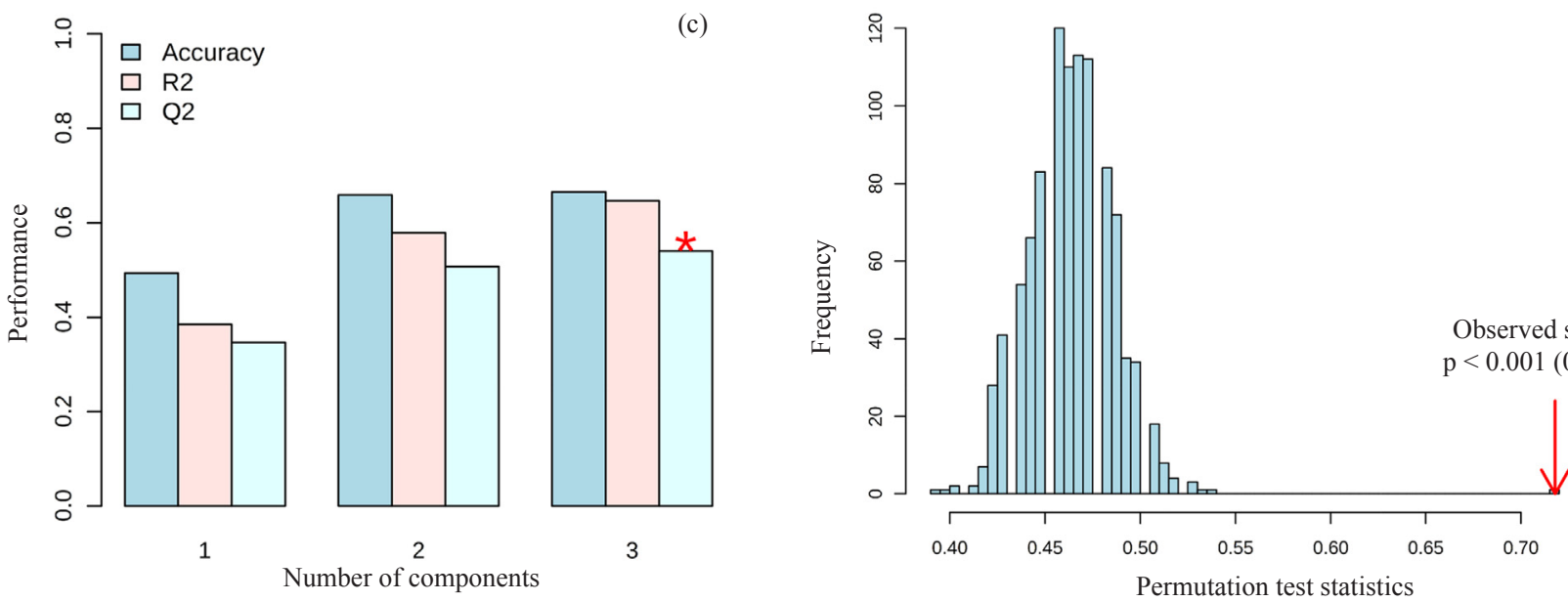

(d)

Fig. 5. PLS-DA of the volatile fingerprinting of apple ciders from different island geographical regions ( $\mathrm{n}=3$ for each data point). a) score scatter plot, b) VIP scores, c) 10-fold cross-validation performance and d) model validation by permutation test based on 1000 permutations of VOCs obtained by GC-qMS of apple cider samples (peak number attribution is shown in Table 3). CAL - Calheta, CL - Câmara de Lobos, SANT - Santana, SC - Santa Cruz, SV - São Vicente.

4-ethyl phenol $(46.4 \pm 2.79$ and $49.9 \pm 3.98 \mu \mathrm{g} / \mathrm{L})$ were the most abundant, with FO of $100 \%$. The OT determined in water $/ 10 \%$ ethanol solution at $\mathrm{pH} 3.2$ for 4-ethyl guaiacol and 4-ethyl phenol is 33 and $440 \mu \mathrm{g} / \mathrm{L}$ (Nikfardjam, May, \& Tschiersch, 2009), respectively. Their presence imparts horsy, barnyard, smoky and medicinal aromatic odours when present at concentration above their OTs. Specific case is the apple cider produced in Santa Cruz (A033_19), since the relative concentrations of the above mentioned volatile phenols were above their OTs $(333 \pm 25.1$ and $489 \pm 41.3 \mu \mathrm{g} / \mathrm{L}$ for 4-ethyl guaiacol and 4-ethyl phenol, respectively). Moreover, the relative concentration of 4ethyl guaiacol was above its OT in $29 \%$ of apple ciders analysed. In addition, 5-methyl guaiacol was only found in apple ciders produced in Câmara de Lobos (FO of 5\%) and Calheta (FO of 40\%), whereas 4vinylguaiacol was not detected in apple ciders from Santa Cruz.

In summary, the mean relative concentration of VOCs identified in apple ciders by geographical regions is showed in Fig. 2. As can be observed, apple ciders from São Vicente seem to be the poorest in all chemical families identified, which may be a result of the mean annual temperature (Table 1) reported for this geographical region. According to Ubalde, Sort, Zayas, and Poch (2010) high temperature affects the fruit development and, consequently accumulation of compounds. On the other hand, apple ciders from Câmara de Lobos seem to be the richest in esters $(504 \pm 20.7 \mu \mathrm{g} / \mathrm{L})$, alcohols $(424 \pm 10.5 \mu \mathrm{g} / \mathrm{L})$ and $\mathrm{C}_{13}$ norisoprenoids $(3.74 \pm 0.45 \mu \mathrm{g} / \mathrm{L})$, whereas apple ciders from Santa Cruz have highest moiety of volatile phenols $(205 \pm 20.6 \mu \mathrm{g} / \mathrm{L})$, acids $(159 \pm 6.92 \mu \mathrm{g} / \mathrm{L})$ and carbonyl compounds $(25.2 \pm 1.49 \mu \mathrm{g} /$ $\mathrm{L})$. Moreover, related to terpenic compounds no remarkable differences were observed between apple ciders produced in Santana $(4.80 \pm 0.97 \mu \mathrm{g} / \mathrm{L})$ and Santa Cruz (4.94 $\pm 1.28 \mu \mathrm{g} / \mathrm{L})$.

Taking into account apple ciders obtained from the same produced from two consecutive years, 2018 and 2019, it was possible to observe that qualitative the profile is quite similar, whereas the semi-quantitative is different (Fig. 3). For esters, alcohols, acids and volatile phenols the total relative average concentrations increased between 2018 and 2019, except for apple ciders produced in São Vicente. This can be explained by the differences observed between samples A026 and A005_19. A possible explanation for the increase in total relative concentration of these chemical families can be supported by the lower mean annual precipitation observed in 2019 (Table 1). A similar pattern was reported by Ubalde et al. (2010) who observed an increase in compound accumulation as a result of water limitation on skin/pulp ratio in driest year. 

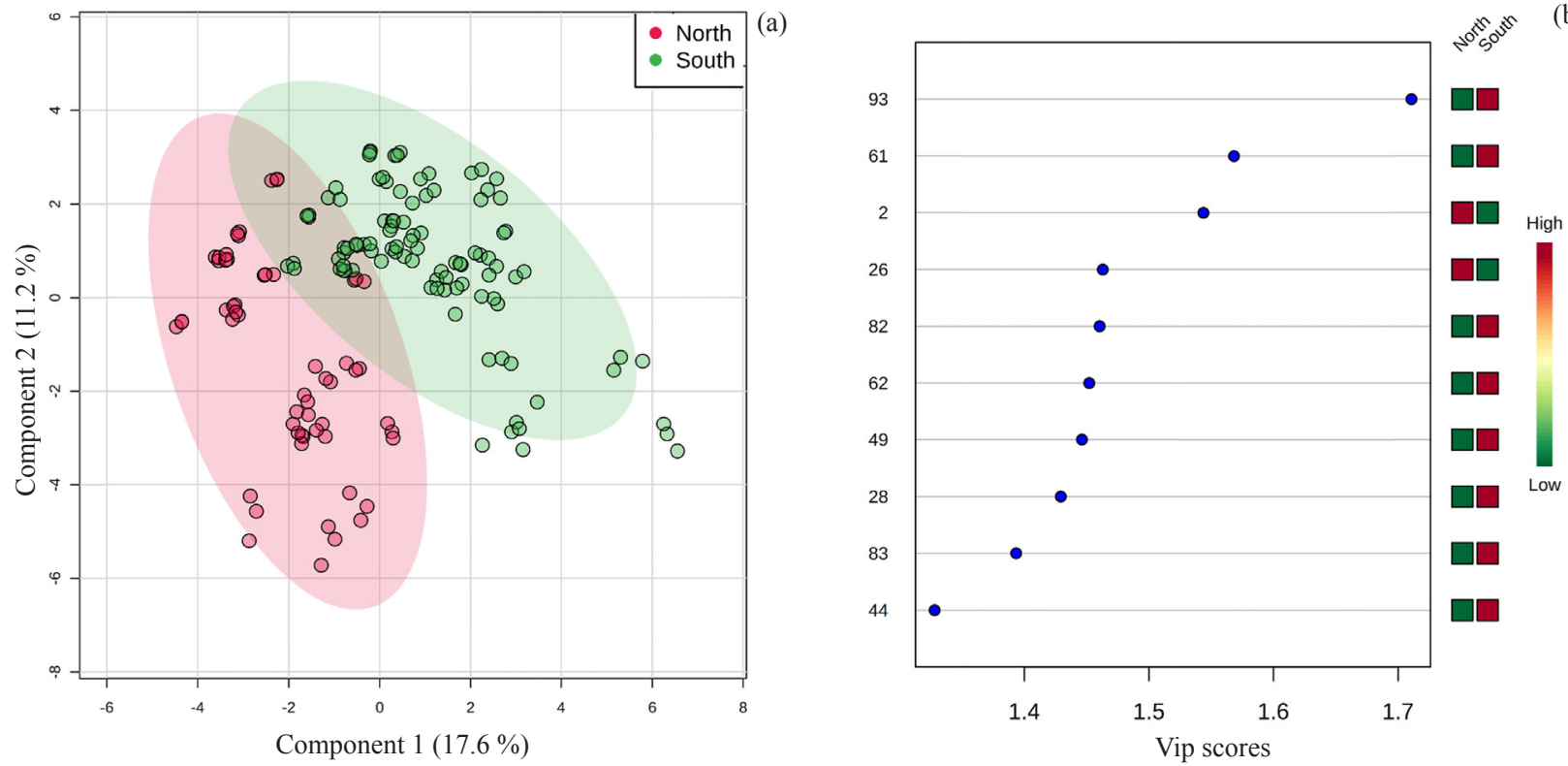

(b)
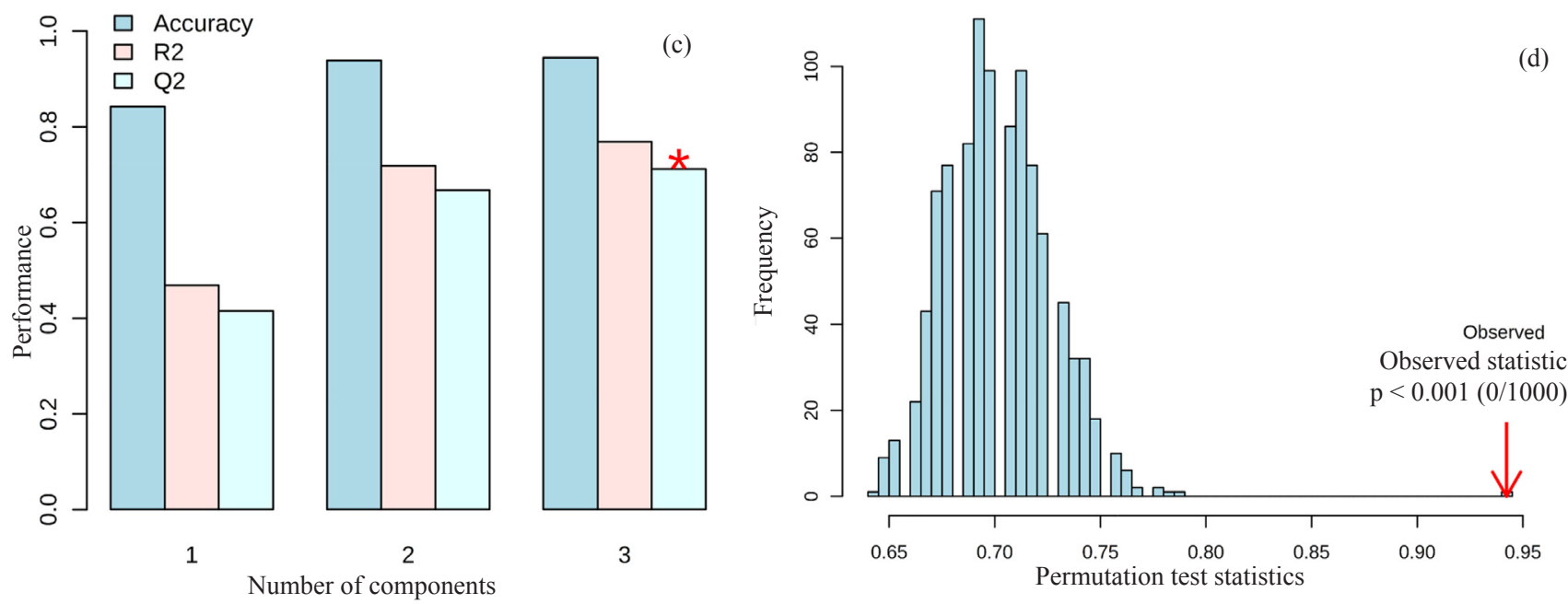

Fig. 6. PLS-DA of the volatile fingerprinting of apple ciders from north and south of Madeira Island ( $\mathrm{n}=3$ for each data point). a) score scatter plot, b) VIP scores, c) 10 -fold cross-validation performance and d) model validation by permutation test based on 1000 permutations of VOCs obtained by GC-qMS of apple cider samples (peak number attribution is shown in Table 3).

\subsection{Statistical analysis}

Statistical analysis was carried out to evaluate the potential to use the volatile fingerprint, as a suitable discriminatory tool for apple ciders, according to their geographical regions, and to guarantee their authenticity and typicity. The one-way ANOVA, followed by post-hoc Tukey's test at $p$-value $<0.001$, was performed to select the VOCs that have statistically significant differences. Forty-three of the identified VOCs showed significant differences among apple ciders from different geographical regions (Fig. 4).

Statistically different VOCs were submitted to PCA analysis that allows to obtain a preliminary overview of similarities and differences among apple cider samples studied. In addition, PCA can show if geographical regions execute a remarkable influence on VOCs relative peak area. To further understand the differences among apple ciders of different geographical origins, a PLS-DA model was developed. Three significant components described 0.6470 of the goodness of fit $\left(\mathrm{R}^{2}=64.70 \%\right)$ and predicted ability of $0.5619\left(\mathrm{Q}^{2}=56.19 \%\right)$ based on crossing-validation. Nevertheless, the score plot (Fig. 5a) did not showed a clear separation of apple ciders based on geographical regions.

Therefore, a novel PLS-DA model was built, and a clear separation was observed between apple ciders produced in the northern and southern regions of Madeira Island (Fig. 6a). Combining the VIP values higher than 1 (Fig. 6b), with the loading plot, 10 VOCs were selected as putative geographical markers for the studied apple ciders. These geographical markers included (Z)-3-hexen-1-ol acetate, 1-octanol, ethyl hexanoate, 1-hexanol, 1,2,3,5-tetramethyl-benzene, ethyl nonanoate, limonene, methyl acetate, ethyl octanoate and isoamyl octanoate). The mean concentration of these VOCs, except methyl acetate, was higher in apple ciders produced in southern region of Madeira Island compared to the samples from north, as can be observed in Fig. 7. In agreement with our findings, (Z)-3-hexen-1-ol acetate, 1-hexanol, and limonene were previously reported as discriminatory geographical markers for apple varieties, with a high prediction accuracy (over 90\%) (Giannetti, Boccacci Mariani, Mannino, \& Marini, 2017). Ethyl octanoate was reported as a discriminatory marker to distinguish Chardonnay wines from other wines with a classification of $74 \%$ (Lukić \& Horvat, 2017). Ethyl hexanoate, ethyl octanoate and 1-hexanol were also reported as putative geographical markers for grapes (Gambetta, 


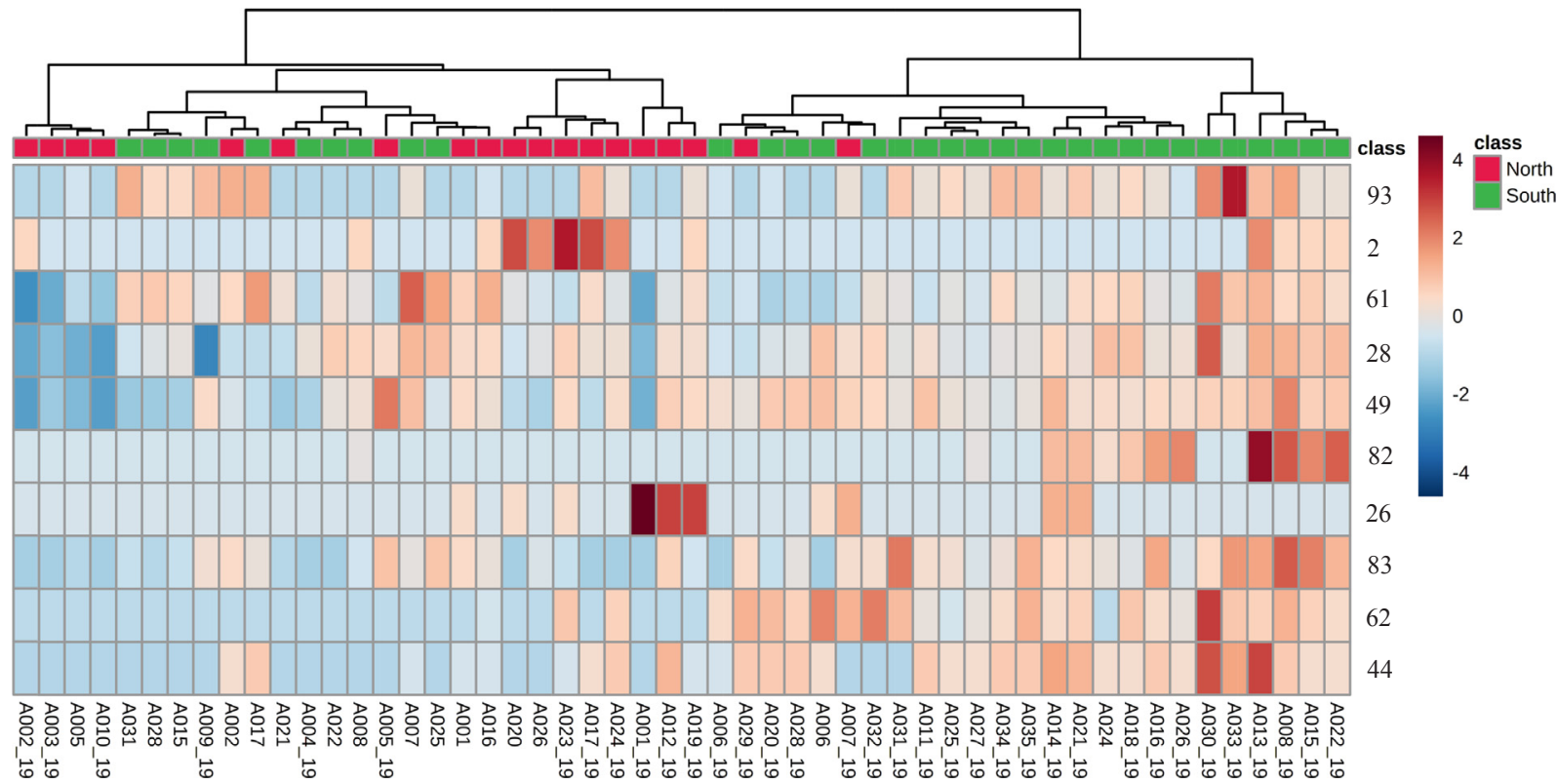

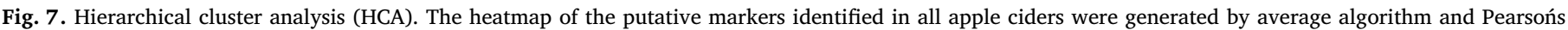
distance analysis (peak number attribution is shown in Table 3).

Cozzolino, Bastian, \& Jeffery, 2017).

To evaluate the robustness of the model, a random permutation test with 1000 permutations was performed with PLS-DA model (Fig. 6c-d). Three significant components described 0.7681 of the goodness of fit $\left(\mathrm{R}^{2}=76.81 \%\right)$ and a predicted ability of $0.7096\left(\mathrm{Q}^{2}=70.96 \%\right)$ based on crossing-validation. Nevertheless, two significant components were sufficient to discriminate samples. The difference between $R^{2}$ and $\mathrm{Q}^{2}$ was lower than $0.3\left(\mathrm{R}^{2}-\mathrm{Q}^{2}=0.06\right)$, which indicated that the model is not over fitted, and showed an acceptable predictive ability for discrimination of apple ciders from different geographical regions.

Fig. 7 displays the resulting dendrogram related to the heat-map constructed by Pearson's correlation, providing instinctive visualization of the dataset, which is often applied to recognize samples or features that are remarkably high or low. An analogous colour tone to the heatmap specifies the area, taking into account the VOCs relative peak area, with VIP values higher than 1 , that a group of samples is similar. The obtained result showed that volatile fingerprint of apple ciders presents some differences among geographical regions. This difference could be a result of climate conditions, altitude and geographic location, which may result in an inhibition of the activity of certain odour related enzymes (Qin et al., 2017). It has been reported that geographic location (e.g., longitude, latitude) and climatic factors (e.g., mean annual temperature) have affect the volatile fingerprint of Fuji apples in the different regions of China (Qin et al., 2017). Furthermore, several studies have reported that grape volatile fingerprint is associated to environmental conditions, namely altitude, soil, topography, macro, meso and microclimate (de Oliveira et al., 2019; De Santis, Frangipane, Brunori, Cirigliano, \& Biasi, 2017; Koundouras, 2018; Perestrelo et al., 2014; Young et al., 2016).

\section{Conclusions}

HS-SPME/GC-qMS data combined with chemometric tools enabled a comprehensive knowledge of the different apple ciders produced in Madeira Island. A total of 143 VOCs were identified in the investigated apple ciders, belonging to different chemical families, and only 28 VOCs from these were common to all apple ciders. Moreover, remarkable differences in terms of qualitative and semi-quantitative profile was obtained, which means that geographical origin and climatic conditions (e.g., precipitation and temperature) have a significant impact on volatile fingerprint. The obtained results revealed that a combination of volatile fingerprint data with chemometric tools provides a powerful tool with a greater ability to accurately discriminate apple ciders from different geographical regions. The developed PLSDA model showed a clear differentiation among apple ciders, giving 6 esters, 2 alcohols and 1 terpenic compound putative geographical markers.

An additional study combining GC-MS with other analytical systems would spread the coverage of apple ciders fingerprint and provide more geographical markers for classification of apple ciders. The results highlight the potential of the used strategy for defining authenticity of analysed apple ciders and, thuse, the possibility of a future recognition of products from Madeira Island as Protected Designation of Origin (PDO) or Traditional Speciality Guaranteed (TSG).

\section{CRediT authorship contribution statement}

António Sousa: Methodology, Writing - original draft. José Vareda: Methodology, Writing - original draft. Regina Pereira: . Catarina Silva: Writing - review \& editing. José S. Câmara: Writing review \& editing. Rosa Perestrelo: Supervision, Writing - review \& editing.

\section{Declaration of Competing Interest}

The authors declare there are no conflict of interest.

\section{Acknowledgements}

This work was supported by FCT-Fundação para a Ciência e a Tecnologia through the CQM Base Fund - UIDB/00674/2020, and Programmatic Fund - UIDP/00674/2020, and by Madeira 14-20 Program, project PROEQUIPRAM - Reforço do Investimento em Equipamentos e Infraestruturas Científicas na RAM (M1420-01-0145FEDER-000008) and by ARDITI - Agência Regional para o Desenvolvimento da Investigação Tecnologia e Inovação, through the project M1420-01-0145-FEDER-000005 - Centro de Química da Madeira - $\mathrm{CQM}^{+}$(Madeira 14-20 Program). 


\section{Appendix A. Supplementary data}

Supplementary data to this article can be found online at https:// doi.org/10.1016/j.foodres.2020.109550.

\section{References}

Andrés-Iglesias, C., Blanco, C. A., García-Serna, J., Pando, V., \& Montero, O. (2016). Volatile compound profiling in commercial lager regular beers and derived alcoholfree beers after dealcoholization by vacuum distillation. Food Analytical Methods, 9(11), 3230-3241.

Antón, M. J., Suárez Valles, B., García Hevia, A., \& Picinelli Lobo, A. (2014). Aromatic profile of ciders by chemical quantitative, gas chromatography-olfactometry, and sensory analysis. Journal of Food Science, 79(1), S92-S99.

Danezis, G. P., Tsagkaris, A. S., Camin, F., Brusic, V., \& Georgiou, C. A. (2016). Food Authentication: Techniques, Trends \& Emerging Approaches. Trends in Analytical Chemistry, 85, 123-132.

de Oliveira, J. B., Egipto, R., Laureano, O., de Castro, R., Pereira, G. E., \& Ricardo-daSilva, J. M. (2019). Climate effects on physicochemical composition of Syrah grapes at low and high altitude sites from tropical grown regions of Brazil. Food Research International, 121, 870-879.

De Santis, D., Frangipane, M. T., Brunori, E., Cirigliano, P., \& Biasi, R. (2017). Biochemical markers for enological potentiality in a grapevine aromatic variety under different soil types. American Journal of Enology and Viticulture, 68(1), 100-111.

Delgado-Pelayo, R., Gallardo-Guerrero, L., \& Hornero-Méndez, D. (2014). Chlorophyll and carotenoid pigments in the peel and flesh of commercial apple fruit varieties. Food Research International, 65(Part B), 272-281.

Gambetta, J. M., Cozzolino, D., Bastian, S. E. P., \& Jeffery, D. W. (2017). Exploring the effects of geographical origin on the chemical composition and quality grading of Vitis vinifera L. Cv. chardonnay grapes. Molecules, 22(2), 218-245.

Giannetti, V., Boccacci Mariani, M., Mannino, P., \& Marini, F. (2017). Volatile fraction analysis by HS-SPME/GC-MS and chemometric modeling for traceability of apples cultivated in the Northeast Italy. Food Control, 78, 215-221.

González-Robles, I. W., \& Cook, D. J. (2016). The impact of maturation on concentrations of key odour active compounds which determine the aroma of tequila. Journal of the Institute of Brewing, 122(3), 369-380.

Koundouras, S. (2018). Environmental and viticultural effects on grape composition and wine sensory properties. Elements, 14(3), 173-178.

Lachowicz, Oszmiański, Uździcka, \& Chmielewska. (2019). The influence of yeast strain, $\beta$-cyclodextrin, and storage time on concentrations of phytochemical components, sensory attributes, and antioxidative activity of novel red apple ciders. Molecules, 24(13), 2477. https://doi.org/10.3390/molecules24132477.

Lentz, M. (2018). The Impact of Simple Phenolic Compounds on Beer Aroma and Flavor. Fermentation, 4(1), 20-33.

Lobo, A. P., Antón-Díaz, M. J., Alonso, J. J. M., \& Valles, B. S. (2016). Characterization of Spanish ciders by means of chemical and olfactometric profiles and chemometrics. Food Chemistry, 213, 505-513.

Lukić, I., \& Horvat, I. (2017). Differentiation of commercial PDO wines produced in Istria (Croatia) according to variety and harvest year based on HS-SPME-GC/MS volatile aroma compounds profiling. Food Technology and Biotechnology, 55(1), 95-108.

Mangas, J., González, M., Rodríguez, R., \& Blanco, D. (1996). Solid-phase extraction and determination of trace aroma and flavour components in cider by GC-MS. Chromatographia, 42(1-2), 101-105.

Medina, S., Perestrelo, R., Santos, R., Pereira, R., \& Câmara, J. S. (2019). Differential volatile organic compounds signatures of apple juices from Madeira Island according to variety and geographical origin. Microchemical Journal, 150, 104094.

Mestre, M. V., Maturano, Y. P., Gallardo, C., Combina, M., Mercado, L., Toro, M. E., ... Dellacassa, E. (2019). Impact on sensory and aromatic profile of low ethanol Malbec wines fermented by sequential culture of Hanseniaspora uvarum and Saccharomyces cerevisiae native yeasts. Fermentation, 5(3), 65. https://doi.org/10.3390/ fermentation 5030065 .
Nešpor, J., Karabín, M., Štulíková, K., \& Dostálek, P. (2019). An HS-SPME-GC-MS method for profiling volatile compounds as related to technology used in cider production. Molecules, 24(11), 2117-2131.

Nicolini, G., Román, T., Carlin, S., Malacarne, M., Nardin, T., Bertoldi, D., \& Larcher, R. (2018). Characterisation of single-variety still ciders produced with dessert apples in the Italian Alps. Journal of the Institute of Brewing, 124, 457-466.

Nikfardjam, M. P., May, B., \& Tschiersch, C. (2009). 4-Ethylphenol and 4-ethylguaiacol contents in bottled wines from the German "Württemberg" region. European Food Research and Technology, 230(2), 333-341. https://doi.org/10.1007/s00217-0091174-1.

Pello-Palma, J., González-Álvarez, J., Gutiérrez-Álvarez, M. D., Dapena de la Fuente, E., Mangas-Alonso, J. J., Méndez-Sánchez, D., ... Arias-Abrodo, P. (2017). Determination of volatile compounds in cider apple juices using a covalently bonded ionic liquid coating as the stationary phase in gas chromatography. Analytical and Bioanalytical Chemistry, 409(11), 3033-3041.

Perestrelo, R., Barros, A. S. A. S., Rocha, S. M., \& Câmara, J. S. (2014). Establishment of the varietal profile of Vitis vinifera L. grape varieties from different geographical regions based on HS-SPME/GC-qMS combined with chemometric tools. Microchemical Journal, 116, 107-117.

Perestrelo, R., Silva, C., \& Câmara, J. S. (2019). Madeira wine volatile profile. A platform to establish madeira wine aroma descriptors. Molecules, 24, 3028-3045.

Perestrelo, R., Silva, C. L., Silva, P., Medina, S., Pereira, R., \& Câmara, J. S. (2019). Untargeted fingerprinting of cider volatiles from different geographical regions by HS-SPME/GC-MS. Microchemical Journal, 148, 643-651.

Picinelli, A., Suárez, B., Moreno, J., Rodríguez, R., Caso-García, L. M., \& Mangas, J. J. (2000). Chemical characterization of Asturian cider. Journal of Agricultural and Food Chemistry, 48(9), 3997-4002.

Pizarro, C., Pérez-del-Notario, N., \& González-Sáiz, J. (2009). Headspace solid-phase microextraction for direct determination of volatile phenols in cider. J. Sep. Science, 32(21), 3746-3754.

Qin, L., Wei, Q. P., Kang, W. H., Zhang, Q., Sun, J., \& Liu, S. Z. (2017). Comparison of volatile compounds in "Fuji" apples in the different regions in China. Food Science and Technology Research, 23(1), 79-89.

Qin, Z., Petersen, M. A., \& Bredie, W. L. P. (2018). Flavor profiling of apple ciders from the UK and Scandinavian region. Food Research International, 105, 713-723.

Sun, Z. G., Wang, M. Q., Wang, Y. P., Xing, S., Hong, K. Q., Chen, Y. F., ... Xiao, D. G. (2019). Identification by comparative transcriptomics of core regulatory genes for higher alcohol production in a top-fermenting yeast at different temperatures in beer fermentation. Applied Microbiology and Biotechnology, 103(12), 4917-4929.

Tesnière, C. (2019, October 1). Importance and role of lipids in wine yeast fermentation. Applied Microbiology and Biotechnology. Springer Verlag.

Ubalde, J. M., Sort, X., Zayas, A., \& Poch, R. M. (2010). Effects of soil and climatic conditions on grape ripening and wine quality of Cabernet Sauvignon. Journal of Wine Research, 21(1), 1-17. https://doi.org/10.1080/09571264.2010.495851.

Uthurry, C. A., Susca, M. J., Fontanini, J. M., Gresia, J. A., Bezic, C. A., Caponi, A. M., \& Franchi, M. L. (2019). Physicochemical and sensorial characterisation of Argentine ciders. Journal of the Institute of Brewing, 125(4), 433-442.

van Den Dool, H., \& Dec. Kratz, P. (1963). A generalization of the retention index system including linear temperature programmed gas-liquid partition chromatography. Journal of Chromatography A, 11, 463-471.

Vinci, G., Preti, R., Tieri, A., \& Vieri, S. (2012). Authenticity and quality of animal origin food investigated by stable-isotope ratio analysis. J Sci Food Agric, 93(3), 439-448.

Włodarska, K., Pawlak-Lemańska, K., Górecki, T., \& Sikorska, E. (2017). Classification of commercial apple juices based on multivariate analysis of their chemical profiles. International Journal of Food Properties, 20(8), 1773-1785. https://doi.org/10.1080/ 10942912.2016.1219367.

Worley, B., \& Powers, R. (2013). Multivariate analysis in metabolomics. Current. Metabolomics, 1(1), 92-107.

Young, P. R., Eyeghe-Bickong, H. A., du Plessis, K., Alexandersson, E., Jacobson, D. A., Coetzee, Z., ... Vivier, M. A. (2016). Grapevine plasticity in response to an altered microclimate: Sauvignon Blanc modulates specific metabolites in response to increased berry exposure. Plant Physiology, 170(3), 1235-1254. Retrieved from http:// www.ncbi.nlm.nih.gov/pubmed/26628747. 